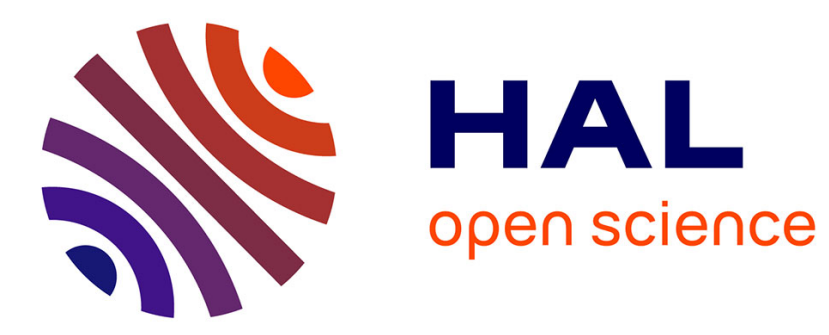

\title{
Photocycloadditions of Arenes Derived from Lignin
}

Arthur Desvals, Stéphane A Baudron, Véronique Bulach, Norbert Hoffmann

\section{To cite this version:}

Arthur Desvals, Stéphane A Baudron, Véronique Bulach, Norbert Hoffmann. Photocycloadditions of Arenes Derived from Lignin. Journal of Organic Chemistry, 2021, 86 (19), pp.13310-13321. 10.1021/acs.joc.1c01361 . hal-03362349

\section{HAL Id: hal-03362349 \\ https://hal.science/hal-03362349}

Submitted on 1 Oct 2021

HAL is a multi-disciplinary open access archive for the deposit and dissemination of scientific research documents, whether they are published or not. The documents may come from teaching and research institutions in France or abroad, or from public or private research centers.
L'archive ouverte pluridisciplinaire HAL, est destinée au dépôt et à la diffusion de documents scientifiques de niveau recherche, publiés ou non, émanant des établissements d'enseignement et de recherche français ou étrangers, des laboratoires publics ou privés. 


\section{Photocycloadditions of Phenols Derived from Lignin}

Arthur Desvals ${ }^{1)}$, Stéphane A. Baudron ${ }^{2)}$, Véronique Bulach ${ }^{2)}$, Norbert Hoffmann ${ }^{* 1)}$

1) CNRS, Université de Reims Champagne-Ardenne, ICMR, Equipe de Photochimie, UFR Sciences,

B.P. 1039,51687 Reims, France

e-mail: norbert.hoffmann@univ-reims.fr

2) CNRS, CMC UMR 7140, Université de Strasbourg, 4 rue Blaise Pascal, 67000, Strasbourg, France.

\section{Abstract}
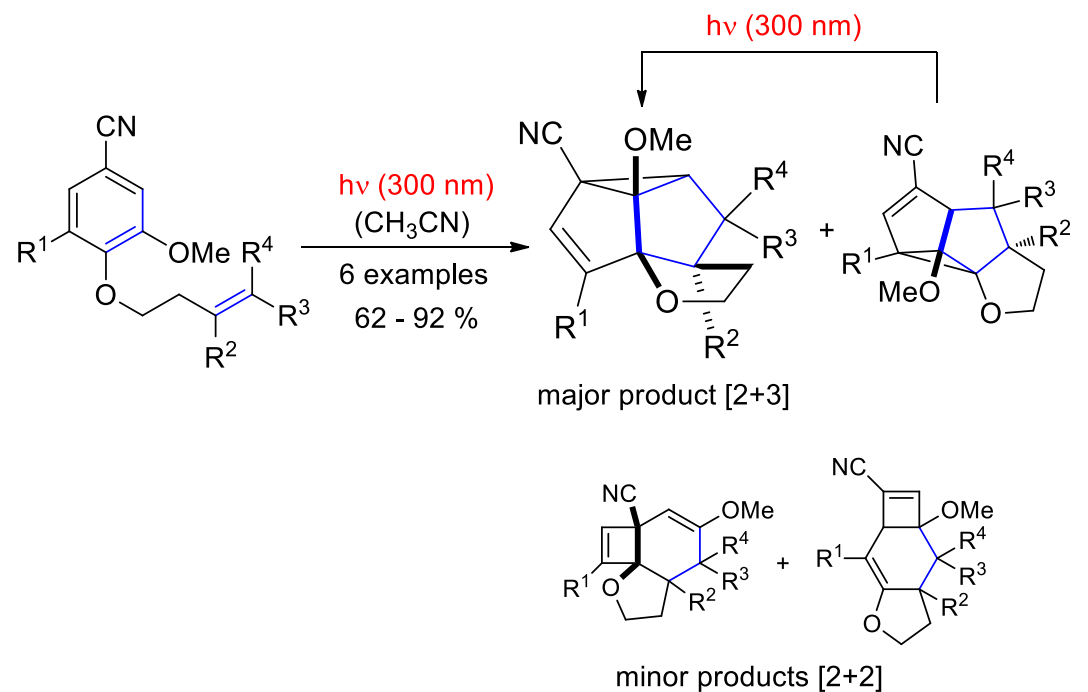

Intramolecular photocycloaddition reactions of 3,4-benzonitriles derived from vanillin with alkenes have been investigated. In contrast to previous reports on photochemical reactions with these compounds, mainly [2+3] cycloaddition has been observed. A competing [2+2] photocycloaddition plays a minor role. Most probably these additions occurs at the singlet state $S_{1}$. In the case of a triplet reaction, a different regioselectivity of the [2+2] cycloaddition would be observed. A linear and an angular [2+3] cycloadduct are formed as major products. The first isomer is transformed in the second one by a photochemical vinyl-cyclopropane rearrangement which increases the selectivity of the reaction. The influence of the substitution pattern on the reactivity and the selectivity has also been investigated. 


\section{Introduction}

Photochemical reactions provide an efficient way to generate a high degree of molecular complexity and diversity. Starting from simple starting compounds, in only one step complex products such as polycyclic compounds are obtained. These reactivities are explained by the fact that photochemically excited compounds have a completely different electronic configuration when compared to the corresponding ground state molecules. ${ }^{1}$ These reactions are therefore particular interesting for being applied to organic synthesis. ${ }^{2,3,4}$ In the case of aromatic compounds or benzene derivatives, the ground state reactions are characterized by a high tendency to regenerate the aromaticity in the final products. Photochemical reactions of such compounds are often characterized by a high tendency to yield non-aromatic final products which is linked to the fact that at the excited state, aromatic character is lost and the compounds become anti-aromatic. ${ }^{5}$ This is the case for photochemical cycloadditions of benzene derivatives with alkenes. ${ }^{6,7,8,9}$ Three cycloaddition modes have been reported: (1) [2+2] or ortho photocycloaddition and consecutive reactions, $(2)[2+3]$ or meta photocycloaddition and $(3)[2+4]$ or para photocycloaddition. Many applications of the meta cycloaddition have been reported. ${ }^{10}$ These reactions are also discussed in the context of dearomatization reactions, with numerous applications in organic synthesis. ${ }^{11}$ It should further be pointed out that these reactions as many other photochemical reactions fulfil requirements of sustainable chemistry. Often no additional chemical activation is needed and the photon is considered as a traceless reagent. ${ }^{6,12}$

Biomass as renewable resources for chemical industry also play a central role in sustainable chemistry. ${ }^{13}$ In this context, Lignin is an important resource for aromatic compounds, in particular phenols. ${ }^{14,15}$ For example, vanillin is produced on industrial scale from lignin. ${ }^{16}$ We became interested in the investigation of photochemical cycloadditions with such derivatives. In intramolecular photocycloadditions between the benzene ring and an alkene, often a competition between the [3+2] and [2+2] mode is observed. ${ }^{17,18}$ Many of these reactions are reversible. In the case of phenol derivatives, these equilibria can be controlled by additives such as acid. ${ }^{19,20,21,22,23}$

We now report on intramolecular photocycloadditions of lignin derived phenols such as vanillin or syringaldehyde carrying an alkene side chain. The influence of additional electron withdrawing and donating substituents such as the nitrile function and alkoxy groups on the competition between [2+2] and [3+2] photocycloaddition is of particular interest. These substituents may also have an influence on the formation of different regio- and stereoisomers. This substitution pattern is typical for many lignin derived phenols. 


\section{Results and Discussions.}

Previous results showed that phenols carrying an alkenyl side chain and an electron-withdrawing substituent such as a carbonyl a carboxyl function or a nitrile substituent undergo preferentially intramolecular [2+2] photocycloaddition which is often followed by thermal or photochemical electrocyclic reactions. ${ }^{8,24,25}$ Two examples have been reported with phenol derivatives carrying a nitrile function as electron withdrawing group (Scheme 1). Compound 1 undergoes [2+2] photocycloaddition leading to the corresponding adduct $\mathbf{A} .{ }^{26} \mathrm{~A}$ thermal electrocyclic reactions, leading to the cyclooctratriene derivative $\mathbf{B}$, followed by a photochemical one yields the final product $\mathbf{2}$. Similar intermolecular reactions have also been observed. ${ }^{27}$ Also when two alkoxy or hydroxy substituents are present on the benzonitrile core, the [2+2] photocycloaddition is favored. When compound $\mathbf{3}$ was irradiated, the tricyclic compound $\mathbf{4}$ was obtained. ${ }^{21}$ Despite the ring constrains the $[2+2]$ cycloaddition takes place in the 1,2-position of the benzonitrile ring (C).<smiles>C=CCCOc1ccc(C#N)cc1</smiles><smiles>C=CCCOc1cc(O)cc(C#N)c1</smiles>

Scheme 1. Selected examples from [2+2] photocycloaddition of phenol derivatives.

In this context, we started our investigations on lignin derived phenol compounds carrying more than one hydroxyl function. We started our investigation with the photochemical transformation of the vanillin derivative 5 . The corresponding [2+2] photocycloadduct 6 was isolated only in low yield. 


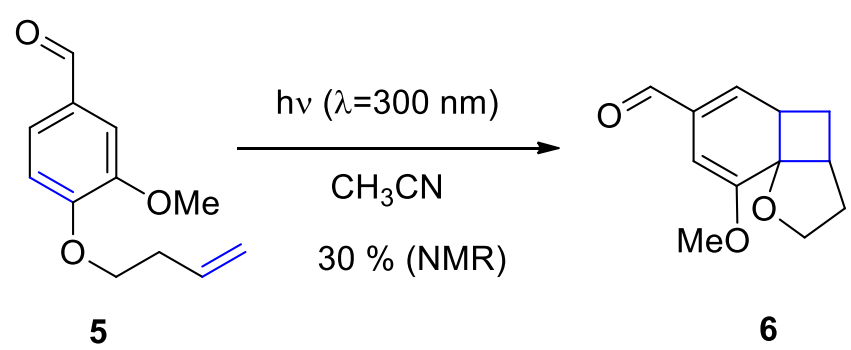

Scheme 2. Unselective [2+2] photocycloaddition of the vanillin derivative $\mathbf{5}$.

As previously mentioned, the nitrile group may be a strong electron withdrawing substituent inducing an efficient [2+2] photocycloaddition in such substrates. Corresponding compounds are easily available. The transformation of aromatic aldehydes $\mathbf{7}$ into nitriles $\mathbf{8}$ was carried out by condensation with hydroxylamine under acidic conditions (Scheme 3). ${ }^{28}$ The final products $\mathbf{1 0}$ have been obtained by 0 alkylation with the corresponding alkenyl bromides or tosylates $9 .{ }^{29}$ Only in case of the fluorinated compound $\mathbf{9}\left(R^{2}, R^{3}, R^{4}=F\right)$, the reaction was less efficient. A competing reaction involving elimination of $\mathrm{HBr}$ at the alkene took place (see experimental section). The yield of this O-alkylation was $20 \%$.<smiles>[R]c1cc(C=O)cc(OC)c1O</smiles>

$7 \quad \mathrm{R}^{1}=\mathrm{H}, \mathrm{OMe}$<smiles>[R]c1cc(C#N)cc(OC)c1O</smiles>

8

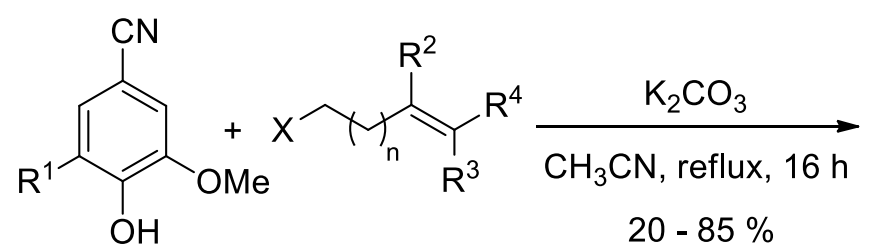

8

$$
9 \mathrm{n}=0,1,2,3
$$$$
\mathrm{X}=\mathrm{Br} \text {, OTs }
$$$$
R^{2}, R^{3}, R^{4}=H, M e, F
$$$$
\mathrm{R}^{2}-\mathrm{R}^{4}=\mathrm{C}_{4} \mathrm{H}_{8}
$$<smiles>[R]C([R])=C([R])[Si]COc1c([R])cc(C#N)cc1OC</smiles>

Scheme 3. Synthesis of the starting compounds of the photochemical reactions.

We started our investigation with photochemical transformations of compound 10a.Compounds 11a and 12a have been isolated as major products resulting from a $[2+3]$ or metacycloaddition (Scheme 4). 
Compound 13a results from a [2+2] or ortho photocycloaddition and was obtained as minor product (Table 1). This result was quite surprising. As pointed out above, we expected that the formation of [2+2] adducts should be highly favored. According to the accepted mechanism for the meta photocycloaddition, the polarized diradical intermediate $\mathbf{D}$ is formed. ${ }^{7,9,10}$ Charge combination according to pathway $a$ leads to the final product 11a and combination according to pathway $b$ yields the final product 12a. Compound 13a results from an initial [2+2] photocycloaddition. According to the accepted mechanism ${ }^{8,9,10}$, the $[2+2]$ adduct $\mathbf{E}$ is transformed in a thermal $(\mathbf{F})$ and a photochemical electrocyclic reaction into the final product 13a. It should be pointed out, that for both the [2+3] (D) and the [2+2] cycloaddition $(\mathbf{E})$, the attack of the alkene to the electronically excited benzonitrile moiety occurs at the same side and close to the methoxy substituent. Depending on the reaction conditions, different product ratios were observed (Table 1). Concerning isolated product yields acetonitrile as solvent (entries 4-8) was comparable to methanol (entries 1-3). The irradiation time and the wavelength had also a significant influence on the results. Most interestingly, compound $12 \mathrm{a}$ could not be isolated when the reaction was carried out at $254 \mathrm{~nm}$ (entries 3-5). When the irradiation was performed at $300 \mathrm{~nm}$, only very prolonged irradiation (entry-7) led to similar results. Under these conditions, it was further observed that the yield of 11a increased. Obviously a part of the latter compound results from a photochemical reaction of 12a. This reaction step is more efficient under irradiation at $254 \mathrm{~nm}$. In acetonitrile compound 13a underwent slowly photochemical decomposition upon prolonged irradiation (compare entries 6 and 7). The decomposition of the photocyclization products is more efficient at irradiation at $254 \mathrm{~nm}$ (entries 3-5). In order to get further information on this competition, we performed a kinetic study. A 49 mM solution was irradiated in a quartz NMR tube at $300 \mathrm{~nm}$ and spectra of the reaction mixture were recorded at different times (Figure 1). We observed that the starting compound 10a was rapidly transformed and compounds 11a, 12a as well as $13 a$ were formed in competition. After consumption of $10 a$ the formation of 13a stopped. No significant decomposition of this compound was observed. After about 30 min, the concentration of $12 \mathrm{a}$ reached a maximum and then decreased while the concentration of 11a continued to increase. Obviously, the linear isomer 12a was transformed into 11a. It was also shown that isolated compound $12 \mathrm{a}$ is transformed into $11 \mathrm{a}$ under these reaction conditions with a yield of $50 \%$. No formation of the starting compound 10a was detected. Therefore, we concluded that this transformation occurs via a vinyl-cyclopropane rearrangement in which intermediate $\mathbf{D}$ is involved. ${ }^{30}$ The corresponding photo-stationary equilibrium is completely shifted to 11 a due to the presence of the $\alpha, \beta$-unsaturated nitrile chromophore in 12a (compare UV spectra in supporting information, Figures S4 and S5). In order to check whether this rearrangement is thermic, we have first irradiated compound 10a in a sealed quartz NMR tube in deuterated methanol at $300 \mathrm{~nm}$ for 100 minutes until full conversion of 10a. The 
reaction mixture was then heated at $70^{\circ} \mathrm{C}$ with a water bath in the dark. No further transformation of 12a into 11a was observed (Figure 2). Under the investigated reaction conditions, the vinyl-cyclopropane rearrangement therefore proceeds photochemically and not thermally.
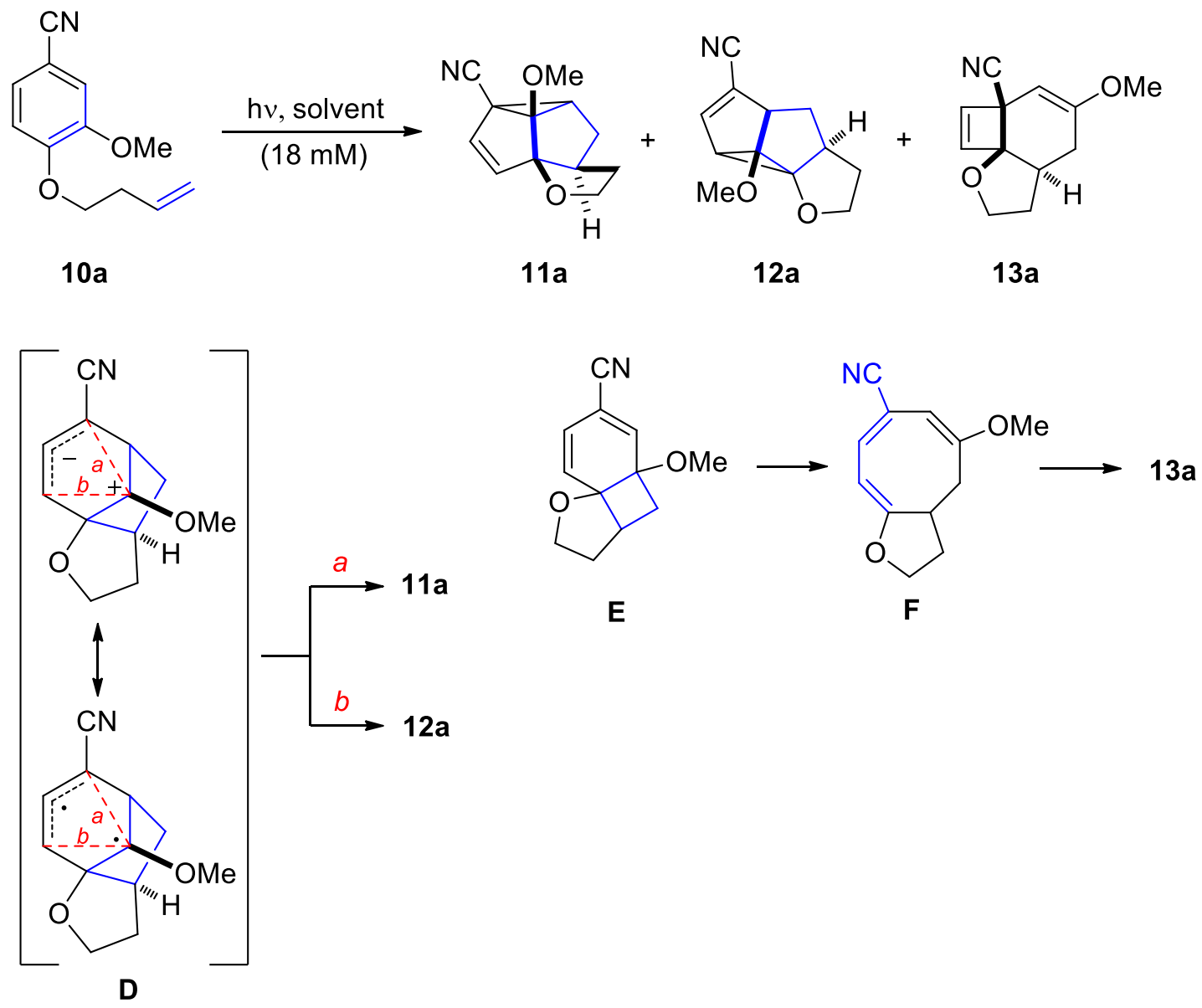

Scheme 4. Highly favored formation of meta photocycloadducts in the intramolecular reaction of compound 10a. 
Table 1. Product yields depending on the reaction conditions (Scheme 1). ${ }^{\text {a) }} 18 \mathrm{mM}$ solutions of 10a were irradiated in quartz tubes in rayonet reactor.

\begin{tabular}{cccccccc}
\hline Entry & $\begin{array}{c}\text { Wavelengh } \\
(\mathrm{nm})\end{array}$ & Solvent $^{\mathrm{a})}$ & $\begin{array}{c}\text { Irradiation } \\
\text { Time }(\mathrm{h})\end{array}$ & Total & 11a & 12a & 13a \\
\hline 1 & 300 & $\mathrm{MeOH}$ & 2 & 84 & 50 & 27 & 7 \\
2 & 300 & $\mathrm{MeOH}$ & 2.5 & 89 & 57 & 25 & 7 \\
3 & 254 & $\mathrm{MeOH}$ & 2.5 & 63 & 61 & 0 & 2 \\
4 & 254 & $\mathrm{MeCN}$ & 2 & 66 & 59 & 0 & 7 \\
5 & 254 & $\mathrm{MeCN}$ & 2.25 & 57 & 54 & 0 & 3 \\
6 & 300 & $\mathrm{MeCN}$ & 2.5 & 92 & 52 & 31 & 9 \\
7 & 300 & $\mathrm{MeCN}$ & 8 & 66 & 61 & 0 & 5 \\
\hline
\end{tabular}

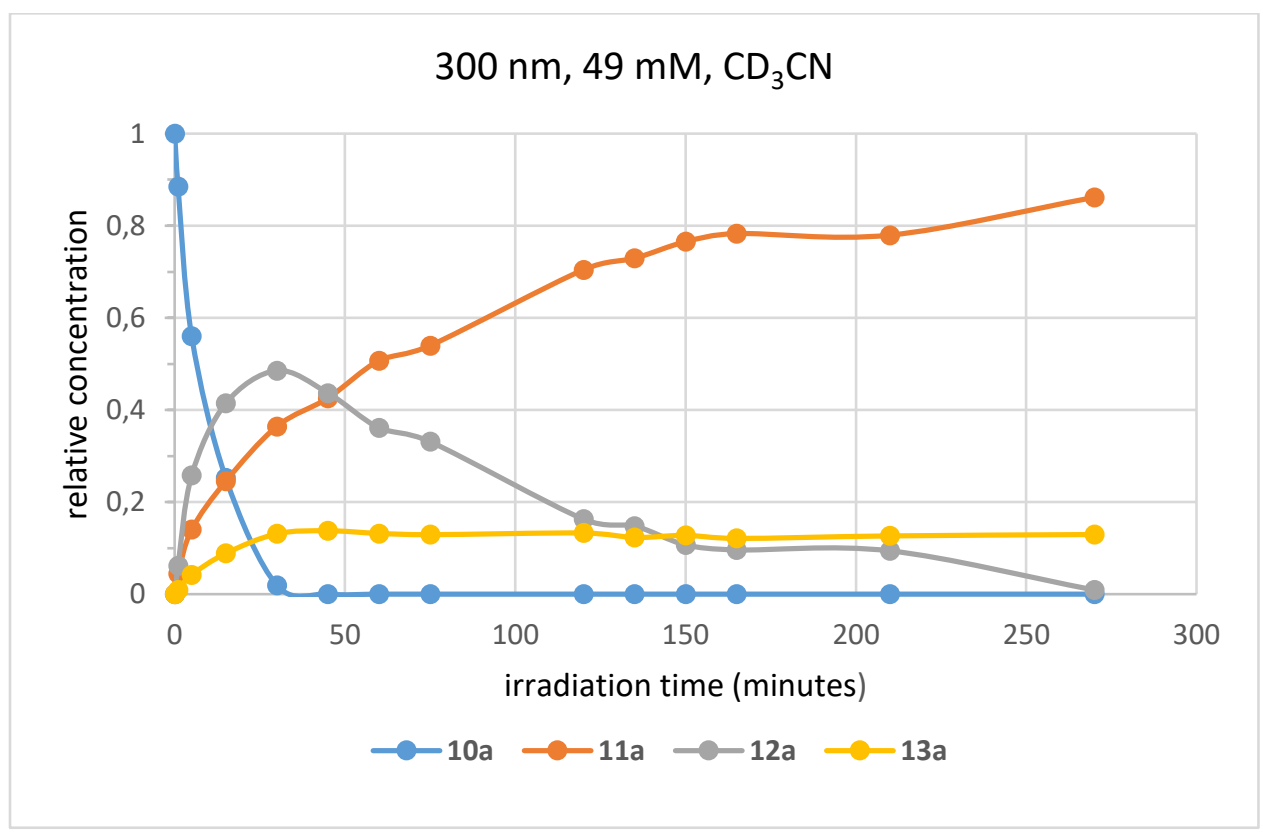

Figure 1. Kinetic of the photochemical transformation of compound 10a. 


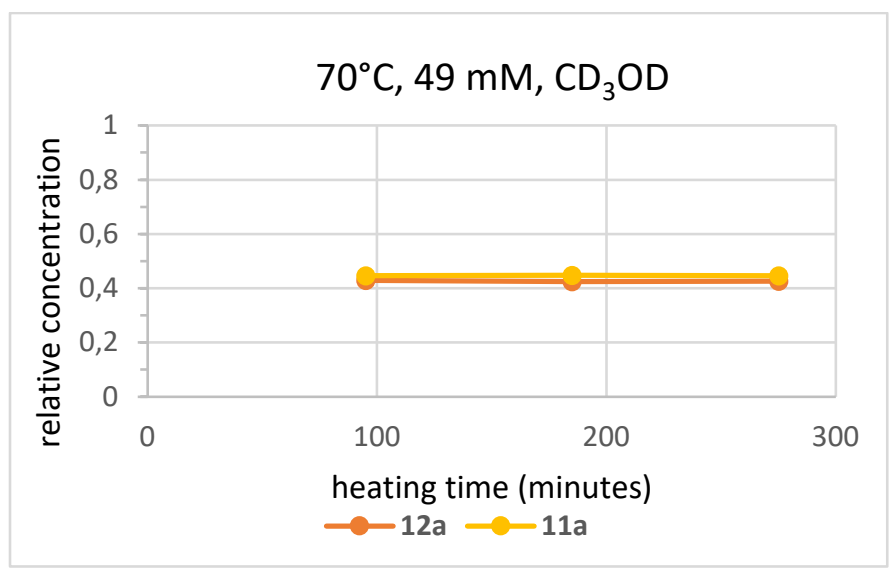

Figure 2. Relative concentrations of $11 \mathrm{a}$ and $12 \mathrm{a}$ in $\mathrm{CD}_{3} \mathrm{OD}$ in NMR tubes heated at $70^{\circ} \mathrm{C}$ in the dark.

First attempts were made to determine scope and limitation of the reaction. In the case of compound 10b carrying a 3-methylbut-3-enyl side chain (Table 2, entry 1), after the reaction time, only the meta adduct $11 \mathbf{b}$ was isolated concomitantly with $13 \mathbf{b}$ the latter resulting from a [2+2] photocycloaddition. After 2.5h of irradiation no starting material was detected and the photostationary equilibrium was established. No linear meta-adduct was detected in the reaction mixture. Compound 10c carrying two methyl groups in the 4 position of the butenyl side chain, was irradiated under the same conditions (entry 2). In this case, a larger proportion of the angular compound $\mathbf{1 3 c}$ and the linear derivative $\mathbf{1 4 c}$ resulting from the dominant $[2+2]$ photocycloaddition was isolated. The linear compound was isolated as a mixture of syn/anti isomers. We have observed that the syn isomer undergoes isomerization to the thermodynamically more stable anti isomer or decomposition in a dark reaction (Scheme 6). In the case of the reaction of $\mathbf{1 0 d}$ (entry 3 ), a smaller portion of the anti isomer of the linear compound $\mathbf{1 4 d}$ (cyclobutene ring and trihydrofuran ring are anti oriented) was isolated as a mixture with 11d. No [2+2] photocycloaddition products were isolated from the transformation of compound $10 \mathrm{e}$ carrying a trifluoroethylene moiety on the side chain (entry 5). In this case, an angular (11e) and a linear (12e) meta cycloaddition product were isolated. In the reaction of the corresponding syringaldehyde derivative $10 \mathrm{f}$ (entry 5), we didn't observe any formation of photo products resulting from a [2+2] cycloaddition. Exclusively, the meta adduct $\mathbf{1 1} \mathbf{f}$ was obtained in good yields. Within this study, it was the most selective reaction.

Further support for the molecular structure was obtained from X-ray structure analysis (Figure 3). Thus the angular regioisomer in compounds $\mathbf{1 1 a , b , c , e ~ w a s ~ c o n f i r m e d . ~ I n ~ t h e ~ s a m e ~ w a y , ~ t h e ~ s t r u c t u r e ~ o f ~ t h e ~}$ angular compound $13 \mathrm{c}$ resulting from a [2+2] photocycloaddition was determined. 
(10b-f

Scheme 5. Intramolecular photocycloaddition of various 3-methoxy-4-butenyloxybenzonitril compounds.

Table 2.

\begin{tabular}{clcccccc}
\hline Entry & \multicolumn{1}{c}{$\mathrm{R}$} & $\begin{array}{c}\text { Irradiation } \\
\text { Time }(\mathrm{h})\end{array}$ & \multicolumn{5}{c}{ Yield (\%) } \\
& & & Total & $\mathbf{1 1}$ & $\mathbf{1 2}$ & $\mathbf{1 3}$ & $\mathbf{1 4}$ \\
\hline 1 & $\mathrm{R}^{1}=\mathrm{H}, \mathrm{R}^{2}=\mathrm{Me}, \mathrm{R}^{3}=\mathrm{R}^{4}=\mathrm{H}(\mathbf{1 0 b})$ & 2.5 & 81 & 71 & & 10 & \\
2 & $\mathrm{R}^{1}=\mathrm{R}^{2}=\mathrm{H}, \mathrm{R}^{3}=\mathrm{R}^{4}=\mathrm{Me}(\mathbf{1 0 c})$ & 2.0 & 68 & 28 & & 14 & $26^{\text {a) }}$ \\
3 & $\mathrm{R}^{1}=\mathrm{H}, \mathrm{R}^{2}-\mathrm{R}^{3}=-\left(\mathrm{CH}_{2}\right)_{4^{-}}, \mathrm{R}^{4}=\mathrm{H}(\mathbf{1 0 d})$ & 2.5 & 68 & 41 & 17 & & $10^{\text {b) }}$ \\
4 & $\mathrm{R}^{1}=\mathrm{H}, \mathrm{R}^{2}=\mathrm{R}^{3}=\mathrm{R}^{4}=\mathrm{F}(\mathbf{1 0 e})$ & 2.7 & 62 & 19 & 43 & & \\
5 & $\mathrm{R}^{1}=\mathrm{OMe}, \mathrm{R}^{2}=\mathrm{R}^{3}=\mathrm{R}^{4}=\mathrm{H}(\mathbf{1 0 f})$ & 1.5 & 63 & 63 & & & \\
\hline
\end{tabular}

a) A 7:3 syn/anti mixture of diastereoisomers was obtained. ${ }^{\text {b) }}$ Only the anti diastereoisomer was formed and isolated as a mixture with $\mathbf{1 1 d}$.

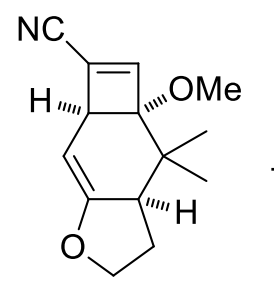

$14 c$

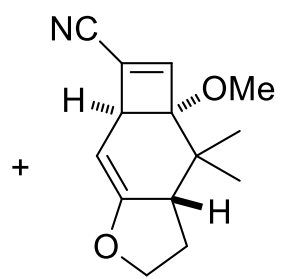

$14 c^{\prime}$
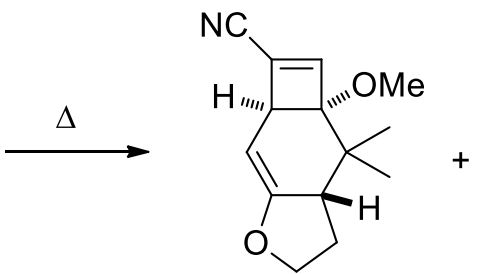

$14 c^{\prime}$<smiles>CO[C@H]1C=C(C#N)[C@H]2CC3=C(CCO3)C(C)(C)[C@@H]21</smiles>

18

Scheme 6. Transformation of $\mathbf{1 4 c}$ in the NMR tube overnight. 


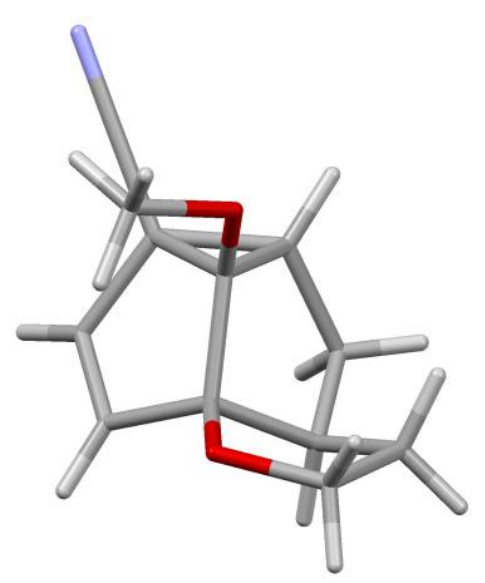

$11 a$

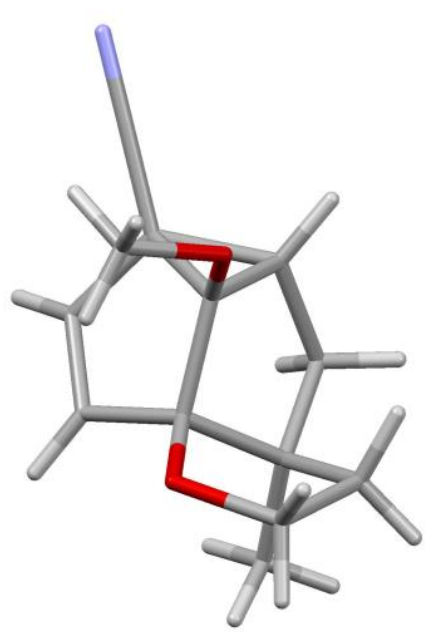

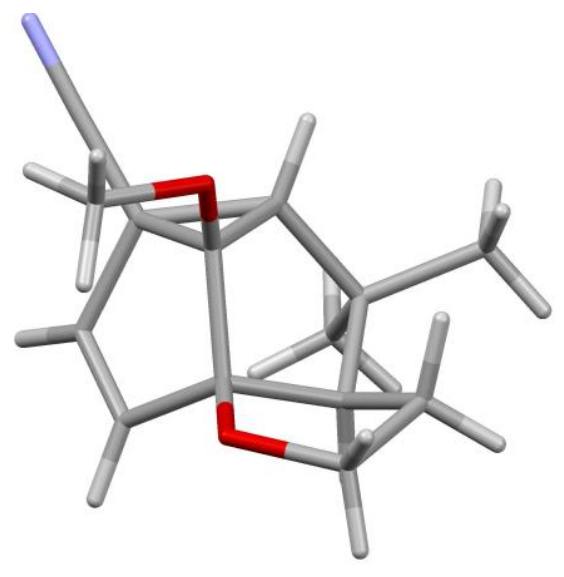

$11 \mathrm{c}$

$11 b$

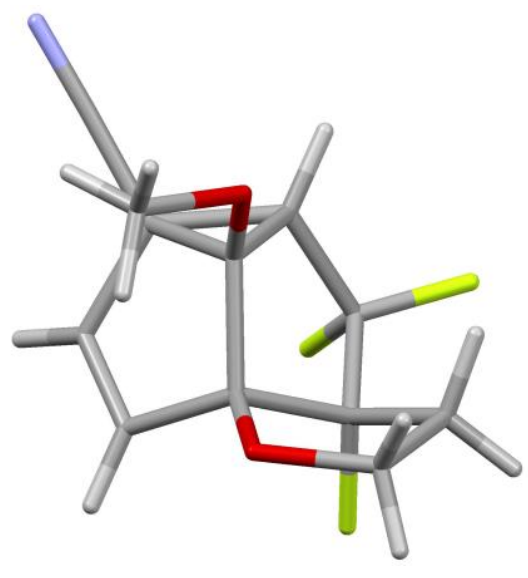

$11 \mathrm{e}$

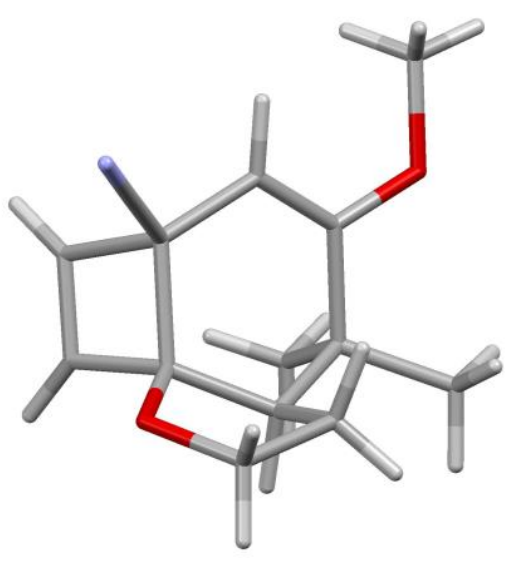

$13 c$

Figure 3. X-ray structure analysis photocycloaddition products $11 \mathbf{a}, \mathbf{b}, \mathbf{c}, \mathbf{e}$ and $\mathbf{1 3 c}$.

We synthesized also vanillin derived benzonitrile compounds with shorter and longer side chaines (Scheme 7). From the reaction of the allyl derivative $10 \mathrm{~g}$, we obtained compound 15 resulting from photo Claisen rearrangement ${ }^{31}$ along with compound 16 . In the case of compounds $10 \mathrm{~h}$ and $10 \mathrm{i}$, only a slow and very unselective photochemical reaction was observed under the herein reported conditions. 


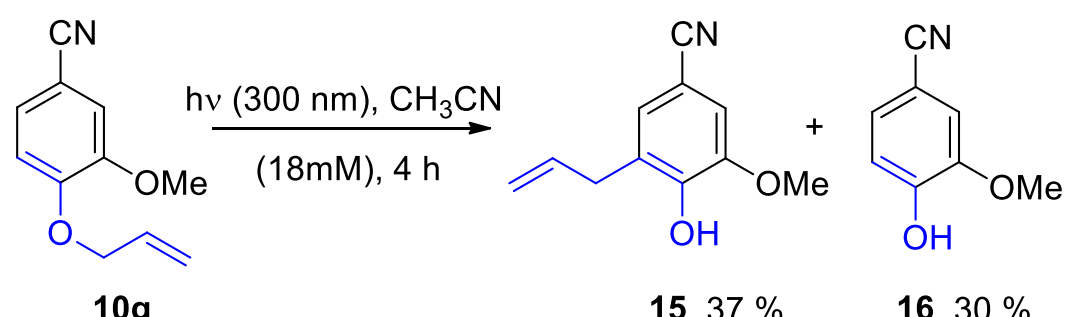<smiles>C=CCCCOc1ccc(C#N)cc1OC</smiles>

$10 \mathrm{~h}$<smiles>COc1cc(C#N)ccc1OCCCCC(F)=C(F)F</smiles>

Scheme 7. Photochemcial reactivity of 3-methoxy-4-butenyloxybenzonitril compounds with shorter or longer side chaines.

Many photocycloadditions of benzene derivatives with alkenes occur at the singlet state. ${ }^{7,8}$ Previously, triplet reactions have been reported with similar compounds carrying carbonyl function as electron withdrawing substituent instead of a nitrile function. ${ }^{24,25}$ In order to check a possible triplet $\left(T_{1}\right)$ reaction with our compounds, we performed the transformation of 10a in the presence of larger amounts of acetone (Scheme 8). Under the irradiation conditions, acetone is electronically excited and transfer its triplet energy to the substrate 10a. Intramolecular [2+2] photocycloaddition takes place and the regio isomer $\mathbf{G}$ is formed. This isomer is different from the isomer $\mathbf{E}$ (Scheme 4) which is formed in a singlet $\left(\mathrm{S}_{1^{-}}\right.$ state) reaction. Such a regioselectivity has previously been observed in reaction of a similar compound which carries an acyl function instead of the nitrile function. ${ }^{32}$ It should further be pointed out that we obtained such a primary photoadduct (6) from the reaction of the vanillin derivative 5 (Scheme 2). Such acetophenone or benzaldehyde derivatives react preferentially at their triplet state. In the present case the intermediate $\mathbf{G}$ undergoes a thermal electrocyclic reaction leading to the cyclooctatriene intermediate $\mathbf{H}$. In a photochemical electrocyclic reaction the final product $\mathbf{1 7 a}$ is formed. This reaction step is also affected by the spin multiplicity. ${ }^{33}$ In the present case, the best yield of 17 a was only $12 \%$. When the ratio acetone/acetonitrile was increased, the reaction became less selective. When the concentration of acetone was diminished, the formation of the singlet products 11a, 12a and 13a was 
detected. We have tested other sensitizers such as benzophenone, 4,4'-dimethoxybenzophenone or thioxanthone without success.
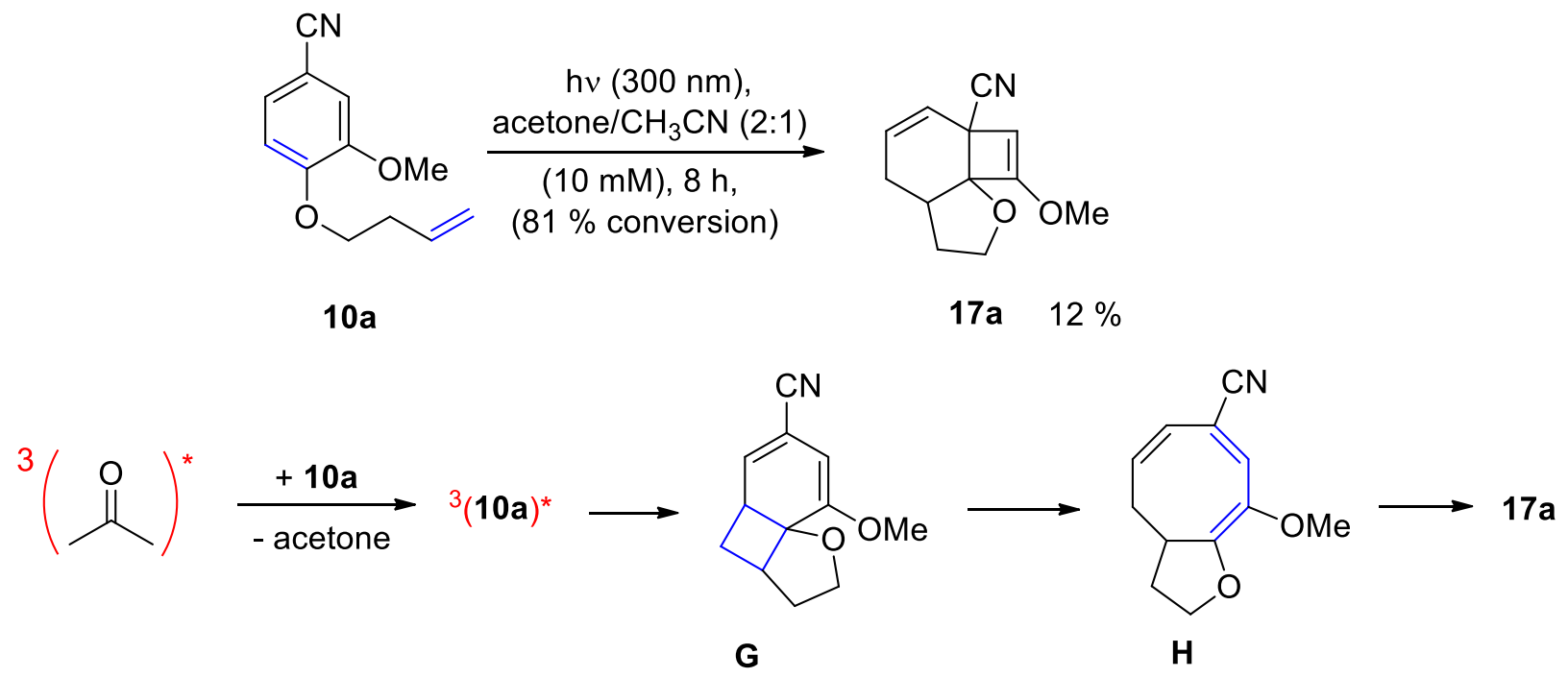

Scheme 8. Triplet sensitized reaction of the 3-methoxy-4-butenyloxybenzonitril derivative 11a.

\section{Conclusion}

We have performed intramolecular photocycloadditions of vanillin derivatives carrying an alkene side chain. In order to increase the efficiency, the aldehyde function was replaced by a nitrile function as electron withdrawing substituent. Often [2+2] photocycloadditions with consecutive rearrangements have been reported with such substrates. In contrast to this, we observed mainly [2+3] photocycloadditions with our substrates. A [2+2] cycloaddition is sometimes observed as side reaction. With a corresponding syringaldehyde derivative only [2+3] cycloaddition was observed. Most probably, the singlet excited state $\left(S_{1}\right)$ is involved in these reactions. The presence of one or two additional alkoxy substituents on the benzene ring favors the $[2+3]$ reaction mode. The influence of the nitrile function favoring the [2+2] cycloaddition is thus reduced. The regioselectivity of the meta cycloadducts (angular versus linear isomers) is increased by an efficient vinyl-cyclopropane rearrangement. The reaction was also carried out under triplet sensitizing conditions with acetone. In this case, only a [2+2] photocycloaddition product with different regioselectivity was isolated in low yields. 


\section{Experimental Section}

General Methods. Photochemical reactions were performed using either a Rayonet RPR-200 photochemical reactor fitted with 16 RPR-3000 Å fluorescent lamps or a Rayonet RPR-100 photochemical reactor fitted with 6 RPR-2537 ̊̊ fluorescent lamps. Melting points were measured in open capillary tubes on a Stuart SMP3 apparatus and are uncorrected. NMR spectra were recorded on Bruker spectrometers operating at $500 \mathrm{MHz}$ or $600 \mathrm{MHz}$ for ${ }^{1} \mathrm{H}, 126 \mathrm{MHz}$ or $151 \mathrm{MHz}$ for ${ }^{13} \mathrm{C}\left\{{ }^{1} \mathrm{H}\right\}$ and $471 \mathrm{MHz}$ for ${ }^{19} \mathrm{~F}$. For ${ }^{1} \mathrm{H}$ NMR spectra, chemical shifts are reported in parts per million (ppm) with reference to tetramethysilane $(\delta=0.00 \mathrm{ppm})$ or residual protonated solvent $\left(\delta=7.26 \mathrm{ppm}\right.$ for $\mathrm{CHCl}_{3,} \delta=1.94 \mathrm{ppm}$ for $\mathrm{CD}_{3} \mathrm{CN}$ ) as internal standard. For ${ }^{13} \mathrm{C}\left\{{ }^{1} \mathrm{H}\right\}$ NMR spectra, chemical shifts are reported in parts per million (ppm) with reference to the deuterated solvent $\left(\delta=77.16 \mathrm{ppm}\right.$ for $\mathrm{CDCl}_{3}, \delta=1.03 \mathrm{ppm}$ for $\mathrm{CD}_{3} \mathrm{CN}$ ) as an internal standard. Splitting patterns for ${ }^{1} \mathrm{H}$ signals are designated as $\mathrm{s}$ (singlet), $\mathrm{d}$ (doublet), $\mathrm{t}$ (triplet), $\mathrm{q}$ (quartet), m (multiplet), br. (broad), or virt. (virtual) and coupling constants $(J)$ are reported in $\mathrm{Hz}$. Structural assignments were made with additional information from gCOSY, gHSQC, and gHMBC experiments. Infrared (IR) spectra were recorded for all samples on a FT-IR Perkin Elmer Spectrum Two using an ATR diamond accessory, maximum absorbance $(v)$ are given in $\mathrm{cm}^{-1}$. UV absorption spectra were recorded with a UVKON 941 PLUS (KONTRON Instruments) in MeCN using $1 \mathrm{~cm}$ quartz cuvettes. Highresolution mass spectrometry (HR-MS) data were recorded using a spectrometer equipped with an electrospray ionization source (ESI-MS) in positive mode associated with a TOF analyzer. MeCN was purchased from Carlo Erba (HPLC quality) and used as received. Organic extracts were dried over magnesium sulfate (VWR Chemicals, 98\%) before evaporation under reduced pressure. Column chromatography was performed on silica gel columns (40-63 $\mu \mathrm{m})$. Analytical thin-layer chromatography was carried out with Kieselgel 60F254 plates from Merck. Retention factors ( $R f$ ) are given for such TLC analyses. Vanillin, Syringaldehyde, Allyl bromide, 4-bromo-1-butene, 3-Methyl-3-buten-1-ol, 5-bromo-1pentene, 5-bromo-2-methyl-2-pentene, 4-bromo-1,1,2-trifluoro-1-butene, 6-bromo-1,1,2-trifluoro-1-

hexene, and 1-Cyclohexene-1-acetic acid were purchased from Merck. Vanillonitrile, ${ }^{28}$ Syringonitrile, ${ }^{34} 3$ Buten-1-ol, 3-methyl-1-(4-methylbenzenesulfonate) ${ }^{35}$ and 2-(1-Cyclohexen-1-yl)ethanol tosylate ${ }^{36}$ were prepared according to the literature, and their spectroscopic data are in agreement with those previously reported. Compounds were crystallized via slow evaporation at room temperature of a petroleum ether/diethyl ether (1:1) solution for $\mathbf{1 1 a}, \mathbf{1 1} \mathbf{c}, \mathbf{1 1 e}, \mathbf{1 3} \mathbf{c}$ and a petroleum ether/ethyl acetate (9:1) solution for $\mathbf{1 1 b}$. X-ray diffraction: data for $\mathbf{1 1 a}, \mathbf{1 1 b}, \mathbf{1 1 c}, \mathbf{1 1 e}$ and $\mathbf{1 3 c}$ were collected at $173(2) \mathrm{K}$ on a Bruker APEX8 CCD Diffractometer equipped with an Oxford Cryosystem liquid N2 device, using a molybdenum microfocus sealed tube generator with mirror-monochromated Mo-K $\alpha$ radiation $(\lambda=$ 
$0.71073 \AA$ A), operated at $50 \mathrm{kV} / 600 \mathrm{~mA}$. The diffraction data were corrected for absorption. Structures were solved using SHELXS-97 and refined by full matrix least-squares on F2 using SHELXL-97. The hydrogen atoms were introduced at calculated positions and not refined (riding model). ${ }^{37}$ They can be obtained free of charge from the Cambridge Crystallographic Data Centre via www.ccdc.cam.ac.uk/datarequest/cif. CCDC N² 2088122, 2087598-2087601.

General procedure for the alkylation. Based on a literature procedure described by Lipshutz et al. ${ }^{29}$ To a solution of aromatic alcohol $(2.0 \mathrm{mmol})$ and $\mathrm{K}_{2} \mathrm{CO}_{3}(5.0 \mathrm{mmol})$ in $\mathrm{MeCN}(8.0 \mathrm{~mL})$ was added the alkenyl halide $(4.0 \mathrm{mmol})$ and the mixture was refluxed with a heat transfer block for $14 \mathrm{~h}$. It was then cool to $22^{\circ} \mathrm{C}$ and the solvent was removed in vacuo. The residue was partitioned between $\mathrm{CH}_{2} \mathrm{Cl}_{2}$ and water and the aqueous layer was extracted with $\mathrm{CH}_{2} \mathrm{Cl}_{2}(2 \times 10 \mathrm{~mL})$. The combined organic extracts were washed with water $(2 \times 10 \mathrm{~mL})$, dried and concentrated in vacuo. The residue was purified by silica gel column chromatography $\left(\mathrm{SiO}_{2}\right.$, solvent, detection mode).

4-(but-3-en-1-yloxy)-3-methoxybenzaldehyde (5). The representative procedure was followed using Vanilline $(0.5 \mathrm{~g}, 3.3 \mathrm{mmol}), \mathrm{K}_{2} \mathrm{CO}_{3}(1.14 \mathrm{~g}, 8.2 \mathrm{mmol})$ and 4-bromo-1-butene $(0.7 \mathrm{~mL}, 6.6$ $\mathrm{mmol}$ ) in $\mathrm{MeCN}(30 \mathrm{~mL})$. Column chromatography ( $\mathrm{SiO}_{2}$, petroleum ether/ethyl acetate $=9: 1, \mathrm{UV}$ ) afforded the product 5 (568 mg, $84 \%$ ) as white crystals. Spectroscopic data are in agreement with those previously reported. ${ }^{38}$

4-(but-3-en-1-yloxy)-3-methoxybenzonitrile (10a). The representative procedure was followed using Vanillonitrile $(2.0 \mathrm{~g}, 13.4 \mathrm{mmol}), \mathrm{K}_{2} \mathrm{CO}_{3}(4.63 \mathrm{~g}, 33.5 \mathrm{mmol})$ and 4-bromo-1-butene $(2.7 \mathrm{~mL}$, $26.8 \mathrm{mmol})$ in $\mathrm{MeCN}(60 \mathrm{~mL})$. Column chromatography $\left(\mathrm{SiO}_{2}\right.$, petroleum ether/ethyl acetate $\left.=9: 1, \mathrm{UV}\right)$ afforded the product $10 \mathrm{a}(2.1 \mathrm{~g}, 77 \%)$ as white crystals. Mp. : $43-44{ }^{\circ} \mathrm{C} . R f=0.35$ (petroleum ether/ethyl acetate = 9:1, UV). ${ }^{1} \mathrm{H} \mathrm{NMR}\left(500 \mathrm{MHz}, \mathrm{CDCl}_{3}\right): \delta 7.25(\mathrm{dd}, J=8.3,1.9 \mathrm{~Hz}, 1 \mathrm{H} ; \mathrm{Ar}-\mathrm{H}), 7.08(\mathrm{~d}, J=1.9 \mathrm{~Hz}, 1 \mathrm{H}$; Ar-H), 6.89 (d, $J=8.3 \mathrm{~Hz}, 1 \mathrm{H} ; \mathrm{Ar}-\mathrm{H}$ ), 5.89 (ddt $J=17.2,10.3,6.7 \mathrm{~Hz}, 1 \mathrm{H} ; \mathrm{CH}$ ), 5.19 (ddt, $J=17.2,1.5,1.4 \mathrm{~Hz}$, $1 \mathrm{H} ; \mathrm{CH}_{2}$ ), 5.14 (ddt, $J=10.3,1.5,1.3 \mathrm{~Hz}, 1 \mathrm{H} ; \mathrm{CH}_{2}$ ), $4.11\left(\mathrm{t}, J=7.0 \mathrm{~Hz}, 2 \mathrm{H} ; \mathrm{CH}_{2}\right), 3.88\left(\mathrm{~s}, 3 \mathrm{H} ; \mathrm{CH}_{3}\right), 2.62$ (tddd, $\left.J=7.0,6.7,1.4,1.3 \mathrm{~Hz}, 2 \mathrm{H} ; \mathrm{CH}_{2}\right) .{ }^{13} \mathrm{C}\left\{{ }^{1} \mathrm{H}\right\} \mathrm{NMR}\left(126 \mathrm{MHz}, \mathrm{CDCl}_{3}\right): \delta 152.5(\mathrm{Cq}), 149.6(\mathrm{Cq}), 133.7(\mathrm{CH}), 126.5$ (CH), $119.4(\mathrm{CN}), 117.8\left(\mathrm{CH}_{2}\right), 114.6(\mathrm{CH}), 112.6(\mathrm{CH}), 104.0(\mathrm{Cq}), 68.4\left(\mathrm{CH}_{2}\right), 56.4\left(\mathrm{CH}_{3}\right), 33.4\left(\mathrm{CH}_{2}\right)$. IR v $\left(\mathrm{cm}^{-}\right.$ $\left.{ }^{1}\right)=3078,3016,2219,1639,1596,1580,993,972,926,919,865$. HRMS (ESI) $: m / z[M+H]^{+}$calcd for $\left[\mathrm{C}_{12} \mathrm{H}_{14} \mathrm{NO}_{2}\right]^{+}: 204.1025$, found : 204.1026 . 
3-methoxy-4-((3-methylbut-3-en-1-yl)oxy)benzonitrile (10b). The representative procedure was followed using Vanillonitrile (0.3 g, $2.1 \mathrm{mmol}), \mathrm{K}_{2} \mathrm{CO}_{3}(0.7 \mathrm{~g}, 5 \mathrm{mmol})$ and 3-Buten-1-ol, 3methyl-, 1-(4-methylbenzenesulfonate) $(1.0 \mathrm{~g}, 4.2 \mathrm{mmol})$ in $\mathrm{MeCN}(15 \mathrm{~mL})$. Column chromatography $\left(\mathrm{SiO}_{2}\right.$, petroleum ether/ethyl acetate $=9: 1, \mathrm{UV})$ afforded the product $10 \mathrm{~b}(388 \mathrm{mg}, 85 \%)$ as white crystals. Mp. : $43-45^{\circ} \mathrm{C} . R f=0.30$ (petroleum ether/ethyl acetate $\left.=9: 1, \mathrm{UV}\right) .{ }^{1} \mathrm{H} \mathrm{NMR}\left(500 \mathrm{MHz}, \mathrm{CDCl}_{3}\right): \delta 7.26(\mathrm{dd}, J=$ 8.3, $1.9 \mathrm{~Hz}, 1 \mathrm{H} ; \mathrm{Ar}-\mathrm{H}$ ), 7.07 (d, J = $1.9 \mathrm{~Hz}, 1 \mathrm{H} ; \mathrm{Ar}-\mathrm{H}), 6.90$ (d, J = 8.3 Hz , 1H; Ar-H), 4.86 (virt. s, $1 \mathrm{H} ; \mathrm{CH}_{2}$ ), 4.80 (virt. s, $1 \mathrm{H} ; \mathrm{CH}_{2}$ ), 4.16 (virt. t, J = $7.2 \mathrm{~Hz}, 2 \mathrm{H} ; \mathrm{CH}_{2}$ ), 3.87 (s, $3 \mathrm{H} ; \mathrm{CH}_{3}$ ), 2.57 (virt. t, J = $7.2 \mathrm{~Hz}, 2 \mathrm{H} ; \mathrm{CH}_{2}$ ), 1.81 (virt. s, 3H; $\left.\mathrm{CH}_{3}\right) .{ }^{13} \mathrm{C}\left\{{ }^{1} \mathrm{H}\right\} \mathrm{NMR}\left(126 \mathrm{MHz}, \mathrm{CDCl}_{3}\right): \delta 152.5(\mathrm{Cq}), 149.6(\mathrm{Cq}), 141.6(\mathrm{Cq}), 126.5(\mathrm{CH}), 119.4$ (CN), $114.6(\mathrm{CH}), 112.6(\mathrm{CH}), 112.5\left(\mathrm{CH}_{2}\right), 104.0(\mathrm{Cq}), 67.8\left(\mathrm{CH}_{2}\right), 56.4\left(\mathrm{CH}_{3}\right), 36.9\left(\mathrm{CH}_{2}\right), 23.0\left(\mathrm{CH}_{3}\right) . \mathrm{IRv}\left(\mathrm{cm}^{-}\right.$ $\left.{ }^{1}\right)=3079,3004,2219,1651,1596,1578,870,866$. HRMS (ESI) : $m / z[M+H]^{+}$calcd for $\left[\mathrm{C}_{13} \mathrm{H}_{16} \mathrm{NO}_{2}\right]^{+}$: 218.1181, found : 218.1183 .

\section{3-methoxy-4-((4-methylpent-3-en-1-yl)oxy)benzonitrile (10c). The representative} procedure was followed using Vanillonitrile $(0.4 \mathrm{~g}, 2.68 \mathrm{mmol}), \mathrm{K}_{2} \mathrm{CO}_{3}(0.9 \mathrm{~g}, 6.7 \mathrm{mmol})$ and 5-bromo-2methyl-2-pentene $(0.39 \mathrm{~mL}, 2.95 \mathrm{mmol})$ in $\mathrm{MeCN}(20 \mathrm{~mL})$. Column chromatography $\left(\mathrm{SiO}_{2}\right.$, petroleum ether/ethyl acetate $=9: 1, \mathrm{UV})$ afforded the product $10 \mathrm{c}(0.3 \mathrm{~g}, 48 \%)$ as white crystals. Mp. : $55^{\circ} \mathrm{C} . R f=0.25$ (petroleum ether/ethyl acetate = 9:1, UV). ${ }^{1} \mathrm{H} \mathrm{NMR}\left(500 \mathrm{MHz}, \mathrm{CDCl}_{3}\right): \delta 7.26(\mathrm{dd}, J=8.3,1.9 \mathrm{~Hz}, 1 \mathrm{H} ; \mathrm{Ar}-\mathrm{H})$, 7.07 (d, J = 1.9 Hz, 1H; Ar-H), 6.88 (d, J = $8.3 \mathrm{~Hz}, 1 \mathrm{H} ; \mathrm{Ar}-\mathrm{H}$ ), 5.18 (tqq, J = 7.2, 1.3, 1.2 Hz, 1H; CH), 4.01 (t, J $=7.3 \mathrm{~Hz}, 2 \mathrm{H} ; \mathrm{CH}_{2}$ ), $3.88\left(\mathrm{~s}, 3 \mathrm{H} ; \mathrm{CH}_{3}\right.$ ), $2.56\left(\mathrm{td}, J=7.3,7.2 \mathrm{~Hz}, 2 \mathrm{H} ; \mathrm{CH}_{2}\right), 1.73\left(\mathrm{~d}, J=1.3 \mathrm{~Hz}, 3 \mathrm{H} ; \mathrm{CH}_{3}\right), 1.66$ (d, $\left.J=1.2 \mathrm{~Hz}, 3 \mathrm{H} ; \mathrm{CH}_{3}\right) .{ }^{13} \mathrm{C}\left\{{ }^{1} \mathrm{H}\right\} \mathrm{NMR}\left(126 \mathrm{MHz}, \mathrm{CDCl}_{3}\right): \delta 152.6(\mathrm{Cq}), 149.6(\mathrm{Cq}), 135.4(\mathrm{Cq}), 126.5(\mathrm{CH}), 119.5$ $(\mathrm{CN}), 118.7(\mathrm{CH}), 114.5(\mathrm{CH}), 112.5(\mathrm{CH}), 103.8(\mathrm{Cq}), 68.7\left(\mathrm{CH}_{2}\right), 56.3\left(\mathrm{CH}_{3}\right), 28.1\left(\mathrm{CH}_{2}\right), 25.9\left(\mathrm{CH}_{3}\right), 18.0$ $\left(\mathrm{CH}_{3}\right) . \mathrm{IR} \vee\left(\mathrm{cm}^{-1}\right)=2972,2922,2223,1685,1582,1512,794,784,765$. HRMS (ESI) : $\mathrm{m} / \mathrm{z}[\mathrm{M}+\mathrm{H}]^{+}$calcd for $\left[\mathrm{C}_{14} \mathrm{H}_{18} \mathrm{NO}_{2}\right]^{+}:$232.1338, found : 232.1337 .

4-(2-(cyclohex-1-en-1-yl)ethoxy)-3-methoxybenzonitrile (10d). The representative procedure was followed using Vanillonitrile $(0.5 \mathrm{~g}, 3.6 \mathrm{mmol}), \mathrm{K}_{2} \mathrm{CO}_{3}(1.2 \mathrm{~g}, 9.0 \mathrm{mmol})$ and 2-(1-Cyclohexen1-yl)ethanol tosylate $(1.0 \mathrm{~g}, 3.6 \mathrm{mmol})$ in $\mathrm{MeCN}(30 \mathrm{~mL})$. Column chromatography $\left(\mathrm{SiO}_{2}\right.$, petroleum ether/ethyl acetate $=9: 1, \mathrm{UV})$ afforded the product $10 \mathrm{~d}(0.4 \mathrm{~g}, 42 \%)$ as white crystals. Mp. : $99{ }^{\circ} \mathrm{C} . R f=$ 0.22 (petroleum ether/ethyl acetate = 9:1, UV). ${ }^{1} \mathrm{H} \mathrm{NMR}\left(500 \mathrm{MHz}, \mathrm{CDCl}_{3}\right): \delta 7.25$ (dd, J=8.4, $1.9 \mathrm{~Hz}, 1 \mathrm{H}$; Ar-H), 7.07 (d, J = 1.9 Hz, 1H; Ar-H), 6.89 (d, J = 8.4 Hz, 1H; Ar-H), 5.52 (virt. s, 1H; CH), 4.11 (t, J = $7.5 \mathrm{~Hz}$, $\left.2 \mathrm{H} ; \mathrm{CH}_{2}\right), 3.87\left(\mathrm{~s}, 3 \mathrm{H} ; \mathrm{CH}_{3}\right), 2.48\left(\mathrm{t}, \mathrm{J}=7.5 \mathrm{~Hz}, 2 \mathrm{H} ; \mathrm{CH}_{2}\right), 1.99\left(\mathrm{~m}, 4 \mathrm{H} ; 2 \mathrm{CH}_{2}\right), 1.63\left(\mathrm{~m}, 2 \mathrm{H} ; \mathrm{CH}_{2}\right), 1.55(\mathrm{~m}, 2 \mathrm{H}$; $\left.\mathrm{CH}_{2}\right) \cdot{ }^{13} \mathrm{C}\left\{{ }^{1} \mathrm{H}\right\}$ NMR (126 MHz, CDCl 3 ): $\delta 152.4(\mathrm{Cq}), 149.4(\mathrm{Cq}), 133.4(\mathrm{Cq}), 126.5(\mathrm{CH}), 124.0(\mathrm{CH}), 119.5$ $(\mathrm{CN}), 114.2(\mathrm{CH}), 112.3(\mathrm{CH}), 103.6(\mathrm{Cq}), 68.2\left(\mathrm{CH}_{2}\right), 56.3\left(\mathrm{CH}_{3}\right), 37.2\left(\mathrm{CH}_{2}\right), 28.8\left(\mathrm{CH}_{2}\right), 25.3\left(\mathrm{CH}_{2}\right), 22.9\left(\mathrm{CH}_{2}\right)$, 
$22.3\left(\mathrm{CH}_{2}\right) . \mathrm{IR} \vee\left(\mathrm{cm}^{-1}\right)=2919,2224,1596,1581,872,799$. HRMS (ESI) : $\mathrm{m} / z[\mathrm{M}+\mathrm{H}]^{+}$calcd for $\left[\mathrm{C}_{16} \mathrm{H}_{20} \mathrm{NO}_{2}\right]^{+}$: 258.1494 , found : 258.1497.

4-(but-3-en-1-yloxy)-3,5-dimethoxybenzonitrile (10f). The representative procedure was followed using Syringonitrile ( $1 \mathrm{~g}, 5.6 \mathrm{mmol}), \mathrm{K}_{2} \mathrm{CO}_{3}(2 \mathrm{~g}, 14 \mathrm{mmol})$ 4-bromo-1-butene (1.2 $\left.\mathrm{mL}, 11 \mathrm{mmol}\right)$ in $\mathrm{MeCN}(30 \mathrm{~mL})$. Column chromatography $\left(\mathrm{SiO}_{2}\right.$, petroleum ether/ethyl acetate $\left.=8.5: 1.5, \mathrm{UV}\right)$ afforded the product $10 f\left(679 \mathrm{mg}, 52 \%\right.$ ) as white crystals. Mp. : $34-36^{\circ} \mathrm{C} . R f=0.29$ (petroleum ether/ethyl acetate = 9:1, UV). ${ }^{1} \mathrm{H}$ NMR (500 MHz, $\mathrm{CDCl}_{3}$ ): $\delta 6.84$ (s, 2H; Ar-H), 5.87 (ddt, J = 17.2, 10.4, $\left.6.7 \mathrm{~Hz}, 1 \mathrm{H} ; \mathrm{CH}\right), 5.13$ (ddt, $J=17.2,1.7,1.6 \mathrm{~Hz}, 1 \mathrm{H} ; \mathrm{CH}_{2}$ ), 5.05 (ddt, $\left.J=10.4,1.7,1.2 \mathrm{~Hz}, 1 \mathrm{H} ; \mathrm{CH}_{2}\right), 4.06\left(\mathrm{t}, J=7.0 \mathrm{~Hz}, 2 \mathrm{H} ; \mathrm{CH}_{2}\right), 3.84$ $\left(\mathrm{s}, 6 \mathrm{H} ; 2 \mathrm{CH}_{3}\right), 2.49$ (tddd, $\left.J=7.0,6.7,1.6,1.2 \mathrm{~Hz}, 2 \mathrm{H} ; \mathrm{CH}_{2}\right) .{ }^{13} \mathrm{C}\left\{{ }^{1} \mathrm{H}\right\} \mathrm{NMR}\left(126 \mathrm{MHz}, \mathrm{CDCl}_{3}\right): \delta 153.8(2 \mathrm{Cq})$, $141.5(\mathrm{Cq}), 134.5(\mathrm{CH}), 119.1(\mathrm{CN}), 116.8\left(\mathrm{CH}_{2}\right), 109.5(2 \mathrm{CH}), 106.7(\mathrm{Cq}), 72.8\left(\mathrm{CH}_{2}\right), 56.4\left(2 \mathrm{CH}_{3}\right), 34.5\left(\mathrm{CH}_{2}\right)$. IR $\vee\left(\mathrm{cm}^{-1}\right)=3071,2937,2227,1579,913,855$. HRMS (ESI) $: \mathrm{m} / z[\mathrm{M}+\mathrm{H}]^{+}$calcd for $\left[\mathrm{C}_{13} \mathrm{H}_{16} \mathrm{NO}_{3}\right]^{+}: 234.1130$, found : 234.1131.

4-(allyloxy)-3-methoxybenzonitrile $(\mathbf{1 0 g})$. The representative procedure was followed using Vanillonitrile $(2.0 \mathrm{~g}, 13 \mathrm{mmol}), \mathrm{K}_{2} \mathrm{CO}_{3}(4.6 \mathrm{~g}, 33 \mathrm{mmol})$ and allyl bromide $(2.3 \mathrm{~mL}, 26 \mathrm{mmol})$ in MeCN $(90$ $\mathrm{mL})$. Column chromatography $\left(\mathrm{SiO}_{2}\right.$, petroleum ether/ethyl acetate $\left.=9: 1, \mathrm{UV}\right)$ afforded the product $10 \mathrm{~g}$ (1.9 g, $78 \%$ ) as white crystals. Mp.: $63^{\circ} \mathrm{C} . R f=0.25$ (petroleum ether/ethyl acetate $\left.=9: 1, \mathrm{UV}\right) .{ }^{1} \mathrm{H}$ NMR (500 MHz, CDCl 3 ): $\delta 7.23(\mathrm{~m}, 1 \mathrm{H} ; \mathrm{Ar}-\mathrm{H}), 7.07(\mathrm{~m}, 1 \mathrm{H} ; \mathrm{Ar}-\mathrm{H}), 6.88(\mathrm{~m}, 1 \mathrm{H} ; \mathrm{Ar}-\mathrm{H}), 6.04(\mathrm{~m}, 1 \mathrm{H} ; \mathrm{CH}), 5.41(\mathrm{~m}$, $\left.1 \mathrm{H} ; \mathrm{CH}_{2}\right), 5.32\left(\mathrm{~m}, 1 \mathrm{H} ; \mathrm{CH}_{2}\right), 4.64\left(\mathrm{~m}, 2 \mathrm{H} ; \mathrm{CH}_{2}\right), 3.88\left(\mathrm{~m}, 3 \mathrm{H} ; \mathrm{CH}_{3}\right) \cdot{ }^{13} \mathrm{C}\left\{{ }^{1} \mathrm{H}\right\} \mathrm{NMR}\left(126 \mathrm{MHz}, \mathrm{CDCl}_{3}\right): \delta 151.9$ (Cq), $149.5(\mathrm{Cq}), 132.1(\mathrm{CH}), 126.2(\mathrm{CH}), 119.2(\mathrm{CN}), 118.9\left(\mathrm{CH}_{2}\right), 114.3(\mathrm{CH}), 112.8(\mathrm{CH}), 104.0(\mathrm{Cq}), 69.8$ $\left(\mathrm{CH}_{2}\right), 56.2\left(\mathrm{CH}_{3}\right) . \mathrm{IR} \vee\left(\mathrm{cm}^{-1}\right)=3021,2984,2222,1596,1582,944,927,863,805$. HRMS (ESI): $\mathrm{m} / \mathrm{z}[\mathrm{M}+\mathrm{H}]^{+}$ calcd for $\left[\mathrm{C}_{11} \mathrm{H}_{12} \mathrm{NO}_{2}\right]^{+}: 190.0868$, found : 190.0867 .

3-methoxy-4-(pent-4-en-1-yloxy)benzonitrile (10h). The representative procedure was followed using Vanillonitrile $(1 \mathrm{~g}, 6.7 \mathrm{mmol}), \mathrm{K}_{2} \mathrm{CO}_{3}(2.32 \mathrm{~g}, 16.8 \mathrm{mmol})$ and 5-bromo-1-pentene $(0.8 \mathrm{~mL}$, $6.7 \mathrm{mmol})$ in $\mathrm{MeCN}(30 \mathrm{~mL})$. Column chromatography $\left(\mathrm{SiO}_{2}\right.$, petroleum ether/ethyl acetate = 9:1, UV) afforded the product $10 \mathrm{~h}(1.2 \mathrm{~g}, 81 \%)$ as white crystals. Mp. : $50-51^{\circ} \mathrm{C} . R f=0.27$ (petroleum ether/ethyl acetate = 9:1, UV). ${ }^{1} \mathrm{H}$ NMR $\left(500 \mathrm{MHz}, \mathrm{CDCl}_{3}\right): \delta 7.26$ (dd, J = 8.3,1.9 Hz, 1H; $\left.\mathrm{Ar}-\mathrm{H}\right), 7.08(\mathrm{~d}, J=1.9 \mathrm{~Hz}, 1 \mathrm{H}$; $\operatorname{Ar}-\mathrm{H}), 6.88(\mathrm{~d}, J=8.3 \mathrm{~Hz}, 1 \mathrm{H} ; \mathrm{Ar}-\mathrm{H}), 5.84(\mathrm{ddt}, J=17,10.3,6.6 \mathrm{~Hz}, 1 \mathrm{H} ; \mathrm{CH}), 5.07$ (ddt, $J=17.1,1.7,1.6 \mathrm{~Hz}$, $1 \mathrm{H} ; \mathrm{CH}_{2}$ ), $5.02\left(\mathrm{ddt}, J=10.3,1.7,1.1 \mathrm{~Hz}, 1 \mathrm{H} ; \mathrm{CH}_{2}\right), 4.06\left(\mathrm{t}, \mathrm{J}=6.7 \mathrm{~Hz}, 2 \mathrm{H} ; \mathrm{CH}_{2}\right), 3.88\left(\mathrm{~s}, 3 \mathrm{H} ; \mathrm{CH}_{3}\right), 2.25(\mathrm{~m}, 2 \mathrm{H}$; $\left.\mathrm{CH}_{2}\right), 1.97\left(\mathrm{~m}, 2 \mathrm{H} ; \mathrm{CH}_{2}\right) .{ }^{13} \mathrm{C}\left\{{ }^{1} \mathrm{H}\right\} \operatorname{NMR}\left(126 \mathrm{MHz}, \mathrm{CDCl}_{3}\right): \delta 152.6(\mathrm{Cq}), 149.6(\mathrm{Cq}), 137.5(\mathrm{CH}), 126.5(\mathrm{CH})$, $119.5(\mathrm{CN}), 115.7\left(\mathrm{CH}_{2}\right), 114.5(\mathrm{CH}), 112.5(\mathrm{CH}), 103.8(\mathrm{Cq}), 68.5\left(\mathrm{CH}_{2}\right), 56.3\left(\mathrm{CH}_{3}\right), 30.0\left(\mathrm{CH}_{2}\right), 28.1\left(\mathrm{CH}_{2}\right) . \mathrm{IR}$ 
$V\left(\mathrm{~cm}^{-1}\right)=3077,2975,2227,1644,1596,1583,994,910,876,799,761$. HRMS (ESI) $: \mathrm{m} / \mathrm{z}[\mathrm{M}+\mathrm{H}]^{+}$calcd for $\left[\mathrm{C}_{13} \mathrm{H}_{16} \mathrm{NO}_{2}\right]^{+}: 218.1181$, found : 218.1183 .

3-methoxy-4-((5,6,6-trifluorohex-5-en-1-yl)oxy)benzonitrile (10i). The representative procedure was followed using Vanillonitrile $(0.7 \mathrm{~g}, 4.7 \mathrm{mmol}), \mathrm{K}_{2} \mathrm{CO}_{3}(1.62 \mathrm{~g}, 11.7 \mathrm{mmol})$ and 6-bromo1,1,2-trifluoro-1-hexene $(1.1 \mathrm{~mL}, 7.0 \mathrm{mmol})$ in $\mathrm{MeCN}(30 \mathrm{~mL})$. Column chromatography $\left(\mathrm{SiO}_{2}\right.$, petroleum ether/ethyl acetate $=9: 1, \mathrm{UV})$ afforded the product $10 \mathrm{i}(880 \mathrm{mg}, 73 \%)$ as white crystals. Mp. : $44-47^{\circ} \mathrm{C} . R f$ $=0.25$ (petroleum ether/ethyl acetate = 9:1, UV). ${ }^{1} \mathrm{H} \mathrm{NMR}\left(500 \mathrm{MHz}, \mathrm{CDCl}_{3}\right): \delta 7.26$ (dd, J = 8.3, $1.9 \mathrm{~Hz}, 1 \mathrm{H}$; $\operatorname{Ar}-\mathrm{H}), 7.08(\mathrm{~d}, J=1.9 \mathrm{~Hz}, 1 \mathrm{H} ; \mathrm{Ar}-\mathrm{H}), 6.88(\mathrm{~d}, J=8.3 \mathrm{~Hz}, 1 \mathrm{H} ; \mathrm{Ar}-\mathrm{H}), 4.07$ (t, J = 6.4 Hz, 2H; CH$)^{2}, 3.88(\mathrm{~s}, 3 \mathrm{H}$; $\left.\mathrm{CH}_{3}\right), 2.38\left(\mathrm{~m}, 2 \mathrm{H} ; \mathrm{CH}_{2}\right), 1.91\left(\mathrm{~m}, 2 \mathrm{H} ; \mathrm{CH}_{2}\right), 1.76\left(\mathrm{~m}, 2 \mathrm{H} ; \mathrm{CH}_{2}\right) .{ }^{13} \mathrm{C}\left\{{ }^{1} \mathrm{H}\right\} \mathrm{NMR}\left(126 \mathrm{MHz}, \mathrm{CDCl}_{3}\right)$ : $\delta 153.4$ (ddd, $J=286.1,272.2,47.4 \mathrm{~Hz} ; \mathrm{CF}_{2}$ ), $152.4(\mathrm{Cq}), 150.0$ (Cq), 128.5 (ddd, $\left.J=234.6,53.4,15.3 \mathrm{~Hz} ; \mathrm{CF}\right), 126.5$ (CH), $119.4(\mathrm{CN}), 114.4(\mathrm{CH}), 112.5(\mathrm{CH}), 104.0(\mathrm{Cq}), 68.6\left(\mathrm{CH}_{2}\right), 56.3\left(\mathrm{CH}_{3}\right), 28.1\left(\mathrm{CH}_{2}\right), 25.4(\mathrm{dd}, J=22.0,1.7 \mathrm{~Hz}$; $\left.\mathrm{CH}_{2}\right), 22.2\left(\mathrm{CH}_{2}\right) .{ }^{19} \mathrm{~F} \mathrm{NMR}\left(471 \mathrm{MHz}, \mathrm{CDCl}_{3}\right): \delta-105.3$ (dd, J=88.7, $\left.32.3 \mathrm{~Hz}, 1 \mathrm{~F} ; \mathrm{C}-\mathrm{F}\right),-124.5$ (dd, $J=114.1$, 88.7 Hz, 1F; C-F $),-174.5$ (dd, J = 114.1, 32.3 Hz, 1F; C-F $)$. IR v $\left(\mathrm{cm}^{-1}\right)=2945,2870,2223,1797,1597,1581$, 990, 877, 817, 797, 737, 693. HRMS (ESI) : $\mathrm{m} / \mathrm{z}[\mathrm{M}+\mathrm{H}]^{+}$calcd for $\left[\mathrm{C}_{14} \mathrm{H}_{15} \mathrm{~F}_{3} \mathrm{NO}_{2}\right]^{+}: 286.1055$, found : 286.1057.

\section{Procedure for the preparation of 3-methoxy-4-((3,4,4-trifluorobut-3-en-1-} yl)oxy)benzonitrile (10e). To a solution of Vanillonitrile $(0.7 \mathrm{~g}, 4.7 \mathrm{mmol})$, dried $\mathrm{Cs}_{2} \mathrm{CO}_{3}(4.59 \mathrm{~g}, 14.1$ $\mathrm{mmol})$, Csl (0.5 g, $1.8 \mathrm{mmol})$ in MeCN (30 mL) was added 1-Butene,4-bromo-1,1,2-trifluoro-, (2.2 mL, 14.1 $\mathrm{mmol}$ ) and the mixture was heated at $70{ }^{\circ} \mathrm{C}$ with a heat transfer block for $14 \mathrm{~h}$. It was then cool to $22{ }^{\circ} \mathrm{C}$ and the solvent was removed in vacuo. The residue was partitioned between $\mathrm{CH}_{2} \mathrm{Cl}_{2}$ and water and the aqueous layer was extracted with $\mathrm{CH}_{2} \mathrm{Cl}_{2}(2 \times 30 \mathrm{~mL})$. The combined organic extracts were washed with water $(2 \times 30 \mathrm{~mL})$, dried and concentrated in vacuo. Column chromatography $\left(\mathrm{SiO}_{2}\right.$, petroleum ether/ethyl acetate $=9: 1, \mathrm{UV})$ afforded the desired product $10 \mathrm{e}(0.24 \mathrm{~g}, 20 \%)$ as white crystals and product 10j $(0.2 \mathrm{~g}$, $18 \%)$ as an amorphous solid.

3-methoxy-4-((3,4,4-trifluorobut-3-en-1-yl)oxy)benzonitrile (10e). Mp. : 54-55 º.

$R f=0.30$ (petroleum ether/ethyl acetate $=9: 1, \mathrm{UV}) .{ }^{1} \mathrm{H} \mathrm{NMR}\left(500 \mathrm{MHz}, \mathrm{CDCl}_{3}\right): \delta 7.26(\mathrm{dd}, J=8.3,1.9 \mathrm{~Hz}$, $1 \mathrm{H} ; \mathrm{Ar}-\mathrm{H}), 7.10(\mathrm{~d}, \mathrm{~J}=1.9 \mathrm{~Hz}, 1 \mathrm{H} ; \mathrm{Ar}-\mathrm{H}), 6.89(\mathrm{~d}, \mathrm{~J}=8.3 \mathrm{~Hz}, 1 \mathrm{H} ; \mathrm{Ar}-\mathrm{H}), 4.23\left(\mathrm{t}, J=6.6 \mathrm{~Hz}, 2 \mathrm{H} ; \mathrm{CH}_{2}\right), 3.88(\mathrm{~s}$, $\left.3 \mathrm{H} ; \mathrm{CH}_{3}\right), 2.85\left(\mathrm{~m}, 2 \mathrm{H} ; \mathrm{CH}_{2}\right) \cdot{ }^{13} \mathrm{C}\left\{{ }^{1} \mathrm{H}\right\} \operatorname{NMR}\left(126 \mathrm{MHz}, \mathrm{CDCl}_{3}\right): \delta 154.2$ (ddd, J = 287.1, 274.2, $45.9 \mathrm{~Hz} ; \mathrm{CF}_{2}$ ), 151.9 (Cq), 149.8 (Cq), $126.4(\mathrm{CH}), 126.1$ (ddd, J = 235.0, 53.6, $17.5 \mathrm{~Hz}$; CF), $119.2(\mathrm{CN}), 114.9(\mathrm{CH}), 113.2$ (CH), 104.9 (Cq), $64.6\left(\mathrm{CH}_{2}\right), 56.4\left(\mathrm{CH}_{3}\right), 26.5\left(\mathrm{dd}, \mathrm{J}=22.0,1.7 \mathrm{~Hz} ; \mathrm{CH}_{2}\right) .{ }^{19} \mathrm{~F} \mathrm{NMR}\left(471 \mathrm{MHz}, \mathrm{CDCl}_{3}\right): \delta-102.5$ 
(dd, J=83.9, 32.9 Hz, 1F; C-F), -122.9 (dd, J = 114.3, 83.9 Hz, 1F; C-F $),-175.3$ (dd, J=114.3, $32.9 \mathrm{~Hz}, 1 \mathrm{~F} ; \mathrm{C}-$ $\left.F_{2}\right)$. IR $\vee\left(\mathrm{cm}^{-1}\right)=3015,2945,2885,2841,2223,1802,1600$. HRMS (ESI) $: \mathrm{m} / \mathrm{z}[\mathrm{M}+\mathrm{H}]^{+}$calcd for $\left[\mathrm{C}_{12} \mathrm{H}_{11} \mathrm{NO}_{2} \mathrm{~F}_{3}\right]^{+}: 258.0742$, found : 258.0744 .

((1,2-difluorobuta-1,3-dien-1-yl)oxy)-3-methoxybenzonitrile (10j). $R f=0.40$ (petroleum ether/ethyl acetate = 9:1, UV). ${ }^{1} \mathrm{H}$ NMR $\left(500 \mathrm{MHz}, \mathrm{CDCl}_{3}\right): \delta 7.26(\mathrm{dd}, J=8.3,1.9 \mathrm{~Hz}, 1 \mathrm{H} ; \mathrm{Ar}-\mathrm{H}), 7.21(\mathrm{~d}, J=$ $1.9 \mathrm{~Hz}, 1 \mathrm{H} ; \mathrm{Ar}-\mathrm{H}), 7.12$ (d, J = 8.3 Hz, 1H; $\mathrm{Ar}-\mathrm{H}), 6.42(\mathrm{~m}, 1 \mathrm{H} ; \mathrm{CH}), 5.54$ (d, J = $\left.17.4 \mathrm{~Hz}, 1 \mathrm{H} ; \mathrm{CH}_{2}\right), 5.29(\mathrm{~m}, 1 \mathrm{H}$; $\left.\mathrm{CH}_{2}\right), 3.93\left(\mathrm{~s}, 3 \mathrm{H} ; \mathrm{CH}_{3}\right) .{ }^{13} \mathrm{C}\left\{{ }^{1} \mathrm{H}\right\} \mathrm{NMR}\left(126 \mathrm{MHz}, \mathrm{CDCl}_{3}\right): \delta 149.8(\mathrm{Cq}), 147.3$ (dd, J = 277.7, 44.5 Hz; CF), 147.1 (Cq), 135.8 (dd, J = 233.8, 56.0 Hz; CF), 125.7 (CH), 121.9 (dd, J = 19.4, 4.8 Hz; CH), 118.3 (CN), 117.1 (CH), $116.1(\mathrm{CH}), 114.8\left(\mathrm{CH}_{2}\right), 108.9(\mathrm{Cq}) 56.6\left(\mathrm{CH}_{3}\right) .{ }^{19} \mathrm{~F} \mathrm{NMR}$ (471 MHz, CDCl 3 ) : $\delta$-120.7 (m, 1F; CF), -173.1 (dd, $J=110.8,26 \mathrm{~Hz}, 1 \mathrm{~F} ; \mathrm{CF}) . \mathrm{IR} \vee\left(\mathrm{cm}^{-1}\right)=2987,2229,1599,913,856,822$. HRMS $(E S I): \mathrm{m} / z[\mathrm{M}+\mathrm{H}]^{+}$calcd for $\left[\mathrm{C}_{12} \mathrm{H}_{10} \mathrm{NO}_{2} \mathrm{~F}_{2}\right]^{+}: 238.0680$, found : 238.0683 .

General procedure for photochemical reactions. In a $20 \mathrm{~mL}$ quartz glass tube $(\varnothing=1.1 \mathrm{~cm}), 4$ (but-3-en-1-yloxy)-3-methoxybenzonitrile $(54 \mathrm{mg}, 0.27 \mathrm{mmol}$ ) was introduced and $\mathrm{MeCN}(15 \mathrm{~mL})$ was added. The tube was equipped with a septum and the mixture was degassed (argon flow, $10 \mathrm{~min}$ ). The mixture was irradiated at $\lambda=300 \mathrm{~nm}$ at room temperature until reaction control (TLC) indicated full conversion of starting material. If full conversion was not reached within $8 \mathrm{~h}$, irradiation was stopped. The solvent was evaporated under reduce pressure, and the crude product was purified by silica gel column chromatography $\left(\mathrm{SiO}_{2}\right.$, solvent, detection mode).

\section{8-methoxy-3,3a,4,4a-tetrahydro-2H-benzo[1,4]cyclobuta[1,2-b]furan-6-}

carbaldehyde (6). The product was prepared following the general procedure for photochemical reactions using 4-(but-3-en-1-yloxy)-3-methoxybenzaldehyde 5 (7 mg, $0.034 \mathrm{mmol}$ ) and $\mathrm{MeCN}$ (15 mL). The tube was irradiated at a wavelength of $300 \mathrm{~nm}$ for 20 minutes. The solvent was evaporated under reduce pressure at $30^{\circ} \mathrm{C}$. Column chromatography (neutral $\mathrm{Al}_{2} \mathrm{O}_{3}$, petroleum ether/ethyl acetate $=9: 1, \mathrm{UV}$ ) afforded product 6 as a yellowish oil. An isolated yield could not be precisely determined because 6 is unstable. Using 1,3-Dimethoxybenzene as internal standard (17 mg, $0.123 \mathrm{mmol}$ added after irradiation) a NMR yield of $30 \%$ was determined for product 6. $R f=0.13$ (petroleum ether/ethyl acetate $=9: 1, \mathrm{UV}$ ). ${ }^{1} \mathrm{H}$ NMR (500 MHz, CDCl $): \delta 9.37(\mathrm{~s}, 1 \mathrm{H} ; \mathrm{CHO}), 6.35(\mathrm{~d}, \mathrm{~J}=5.5 \mathrm{~Hz}, 1 \mathrm{H} ; \mathrm{CH}), 5.62(\mathrm{~s}, 1 \mathrm{H} ; \mathrm{CH}), 4.25(\mathrm{~m}, 2 \mathrm{H}$; $\left.\mathrm{CH}_{2}\right), 3.68\left(\mathrm{~s}, 3 \mathrm{H} ; \mathrm{CH}_{3}\right), 3.41(\mathrm{~m}, 1 \mathrm{H} ; \mathrm{CH}), 3.29(\mathrm{~m}, 1 \mathrm{H} ; \mathrm{CH}), 2.14\left(\mathrm{~m}, 2 \mathrm{H} ; \mathrm{CH}_{2}\right), 1.98\left(\mathrm{~m}, 1 \mathrm{H} ; \mathrm{CH}_{2}\right), 1.78(\mathrm{~m}$, $\left.1 \mathrm{H} ; \mathrm{CH}_{2}\right) .{ }^{13} \mathrm{C}\left\{{ }^{1} \mathrm{H}\right\} \operatorname{NMR}\left(126 \mathrm{MHz}, \mathrm{CDCl}_{3}\right): \delta 191.5(\mathrm{CHO}), 157.1(\mathrm{Cq}), 141.4(\mathrm{CH}), 136.4(\mathrm{Cq}), 90.1(\mathrm{CH}), 82.6$ (Cq), $69.6\left(\mathrm{CH}_{2}\right), 55.7\left(\mathrm{CH}_{3}\right), 48.9(\mathrm{CH}), 41.4(\mathrm{CH}), 33.9\left(\mathrm{CH}_{2}\right), 29.3\left(\mathrm{CH}_{2}\right) . \mathrm{IR} \vee\left(\mathrm{cm}^{-1}\right)=2938,1680,1399,694$. HRMS (ESI) : $m / z[M+H]^{+}$calcd for $\left[\mathrm{C}_{12} \mathrm{H}_{15} \mathrm{O}_{3}\right]^{+}: 207.1021$, found : 207.1020. 
4a1-methoxy-3,3a,4,4a,4a1,4b-hexahydro-2H-cyclopropa[3,4]-pentaleno-[6a,1b]furan-4b-carbonitrile (11a). The product was prepared following the general procedure for photochemical reactions using 4-(but-3-en-1-yloxy)-3-methoxybenzonitrile 10a (54 mg, $0.27 \mathrm{mmol}$ ) and $\mathrm{MeCN}(15 \mathrm{~mL})$. The tube was irradiated at a wavelength of $300 \mathrm{~nm}$ for $8 \mathrm{~h}$. Column chromatography $\left(\mathrm{SiO}_{2}\right.$, petroleum ether/ethyl acetate $\left.=9: 1, \mathrm{KMnO}_{4}\right)$ afforded the product $11 \mathrm{a}(33 \mathrm{mg}, 61 \%)$ as a colorless oil. Product 11a was crystallized as colorless crystals via slow evaporation of a petroleum ether/diethyl ether (1:1) solution at room temperature. Mp. : $90^{\circ} \mathrm{C} . R f=0.25$ (petroleum ether/ethyl acetate $=9: 1$, UV). ${ }^{1} \mathrm{H}$ NMR (500 MHz, CDCl $): \delta 5.78(\mathrm{~d}, J=5.9 \mathrm{~Hz}, 1 \mathrm{H} ; \mathrm{CH}), 5.64$ (d, $\left.J=5.9 \mathrm{~Hz}, 1 \mathrm{H} ; \mathrm{CH}\right), 4.26$ (ddd, $J=$ 9.5, 8.3, 1.7 Hz, $1 \mathrm{H} ; \mathrm{CH}_{2}$ ), 3.97 (ddd, $J=10.5,8.3,6.4 \mathrm{~Hz}, 1 \mathrm{H} ; \mathrm{CH}_{2}$ ), 3.65 (s, 3H; $\mathrm{CH}_{3}$ ), 2.78 (d, J=6.4 Hz, $1 \mathrm{H} ; \mathrm{CH}), 2.41-2.32(\mathrm{~m}, 1 \mathrm{H} ; \mathrm{CH}), 2.06\left(\mathrm{~m}, 1 \mathrm{H} ; \mathrm{CH}_{2}\right), 2.00-1.92\left(\mathrm{~m}, 2 \mathrm{H} ; \mathrm{CH}_{2}\right), 1.56$ (ddd, J = 14.8, 6.4, $1.0 \mathrm{~Hz}$, $\left.1 \mathrm{H} ; \mathrm{CH}_{2}\right) .{ }^{13} \mathrm{C}\left\{{ }^{1} \mathrm{H}\right\} \operatorname{NMR}\left(126 \mathrm{MHz}, \mathrm{CDCl}_{3}\right): \delta 135.1(\mathrm{CH}), 121.7(\mathrm{CH}), 118.2(\mathrm{CN}), 98.2(\mathrm{Cq}), 93.4(\mathrm{Cq}), 71.3$ $\left(\mathrm{CH}_{2}\right), 57.4\left(\mathrm{CH}_{3}\right), 53.9(\mathrm{CH}), 47.4(\mathrm{CH}), 30.5(\mathrm{Cq}), 28.9\left(\mathrm{CH}_{2}\right), 24.2\left(\mathrm{CH}_{2}\right)$. IR v $\left(\mathrm{cm}^{-1}\right)=3028,2967,2225$, 985, 950, 774, 754, 666. HRMS (ESI) : $m / z[\mathrm{M}+\mathrm{H}]^{+}$calcd for $\left[\mathrm{C}_{12} \mathrm{H}_{14} \mathrm{NO}_{2}\right]^{+}: 204.1025$, found : 204.1025.

\section{4a1-methoxy-3a-methyl-3,3a,4,4a,4a1,4b-hexahydro-2H-cyclo-propa-}

$[3,4]$ pentaleno[6a,1-b]furan-4b-carbonitrile (11b). The product was prepared following the

general procedure for photochemical reactions using 3-methoxy-4-((3-methylbut-3-en-1yl)oxy)benzonitrile 10b (58 mg, $0.27 \mathrm{mmol})$ and $\mathrm{MeCN}(15 \mathrm{~mL})$. The tube was irradiated at a wavelength of $300 \mathrm{~nm}$ for $2 \mathrm{~h} 30$. Column chromatography $\left(\mathrm{SiO}_{2}\right.$, petroleum ether/ethyl acetate $\left.=9: 1, \mathrm{KMnO}_{4}\right)$ afforded the product 11b (41 mg, $71 \%$ ) as a colorless oil. Product 11b was crystallized as colorless crystals via slow evaporation of a petroleum ether/ethyl acetate (9:1) solution at room temperature. Mp. : $102{ }^{\circ} \mathrm{C} . R f=0.33$ (petroleum ether/ethyl acetate = 9:1, UV). ${ }^{1} \mathrm{H} \mathrm{NMR}\left(500 \mathrm{MHz}, \mathrm{CDCl}_{3}\right): \delta 5.75(\mathrm{~d}, J=6.0 \mathrm{~Hz}, 1 \mathrm{H} ; \mathrm{CH}), 5.70$ (d, $J=6.0 \mathrm{~Hz}, 1 \mathrm{H} ; \mathrm{CH}$ ), 4.22 (ddd, $J=10.0,8.2,1.5 \mathrm{~Hz}, 1 \mathrm{H} ; \mathrm{CH}_{2}$ ), 4.07 (ddd, $J=10.6,8.2,6.7 \mathrm{~Hz}, 1 \mathrm{H} ; \mathrm{CH}_{2}$ ), $3.68\left(\mathrm{~s}, 3 \mathrm{H} ; \mathrm{CH}_{3}\right), 2.70(\mathrm{~d}, J=6.6 \mathrm{~Hz}, 1 \mathrm{H} ; \mathrm{CH}), 2.34$ (ddd, J=12.2, 10.6, $10.0 \mathrm{~Hz}, 1 \mathrm{H}, \mathrm{CH}_{2}$ ), 2.07 (dd, J = 14.6, $6.6 \mathrm{~Hz}, 1 \mathrm{H} ; \mathrm{CH}_{2}$ ), 1.66 (ddd, J=12.2, 6.7, $\left.1.5 \mathrm{~Hz}, 1 \mathrm{H} ; \mathrm{CH}_{2}\right), 1.27$ (d, J=14.6 Hz, $\left.1 \mathrm{H} ; \mathrm{CH}_{2}\right), 0.92\left(\mathrm{~s}, 3 \mathrm{H} ; \mathrm{CH}_{3}\right)$. ${ }^{13} \mathrm{C}\left\{{ }^{1} \mathrm{H}\right\}$ NMR $\left(126 \mathrm{MHz}, \mathrm{CDCl}_{3}\right): \delta 134.4(\mathrm{CH}), 122.9(\mathrm{CH}), 118.4(\mathrm{CN}), 99.5(\mathrm{Cq}), 94.9(\mathrm{Cq}), 69.2\left(\mathrm{CH}_{2}\right), 60.0$ (Cq), $57.4\left(\mathrm{CH}_{3}\right), 46.0(\mathrm{CH}), 35.0\left(\mathrm{CH}_{2}\right), 32.1\left(\mathrm{CH}_{2}\right), 30.0(\mathrm{Cq}), 20.1\left(\mathrm{CH}_{3}\right) . \mathrm{IR} v\left(\mathrm{~cm}^{-1}\right)=3074,2964,2229,991$, $970,870,797,725$. HRMS (ESI) : $m / z[\mathrm{M}+\mathrm{H}]^{+}$calcd for $\left[\mathrm{C}_{13} \mathrm{H}_{16} \mathrm{NO}_{2}\right]^{+}: 218.1181$, found : 218.1179 .

\section{4a1-methoxy-4,4-dimethyl-3,3a,4,4a,4a1,4b-hexahydro-2H-cyclopropa-}

$[3,4]$ pentaleno[6a,1-b]furan-4b-carbonitrile (11c). The product was prepared following the general procedure for photochemical reactions using 3-methoxy-4-((4-methylpent-3-en-1- 
yl)oxy)benzonitrile 10c (61.7 mg, $0.27 \mathrm{mmol})$ and $\mathrm{MeCN}(15 \mathrm{~mL})$. The tube was irradiated at a wavelength of $300 \mathrm{~nm}$ for $2 \mathrm{~h}$. Column chromatography $\left(\mathrm{SiO}_{2}\right.$, petroleum ether/ethyl acetate $\left.=9: 1, \mathrm{KMnO}_{4}\right)$ afforded the product 11c (17 mg, $28 \%$ ) as a colorless oil. Product 11c was crystallized as colorless crystals via slow evaporation of a petroleum ether/diethyl ether (1:1) solution at room temperature. Mp. : $107^{\circ} \mathrm{C} . R f=0.33$ (petroleum ether/ethyl acetate = 9:1, UV). ${ }^{1} \mathrm{H}$ NMR $\left(500 \mathrm{MHz}, \mathrm{CD}_{3} \mathrm{CN}\right): \delta 5.82(\mathrm{~d}, J=5.8 \mathrm{~Hz}, 1 \mathrm{H} ; \mathrm{CH}), 5.77$ (d, J = 5.8 Hz, 1H; CH), 4.15 (ddd, J = 9.5, 8.2, $1.7 \mathrm{~Hz}, 1 \mathrm{H} ; \mathrm{CH}_{2}$ ), 3.83 (ddd, J=11.4, 8.2, $5.3 \mathrm{~Hz}, 1 \mathrm{H} ; \mathrm{CH}_{2}$ ), 3.57 (s, 3H; $\left.\mathrm{CH}_{3}\right), 2.59(\mathrm{~s}, 1 \mathrm{H} ; \mathrm{CH}), 2.28(\mathrm{dd}, \mathrm{J}=11.8,6.9 \mathrm{~Hz}, 1 \mathrm{H} ; \mathrm{CH}), 2.11\left(\mathrm{~m}, 1 \mathrm{H} ; \mathrm{CH}_{2}\right), 1.89\left(\mathrm{~m}, 1 \mathrm{H} ; \mathrm{CH}_{2}\right), 1.13$ (s, 3H; $\left.\mathrm{CH}_{3}\right), 1.06$ (s, 3H; $\left.\mathrm{CH}_{3}\right) .{ }^{13} \mathrm{C}\left\{{ }^{1} \mathrm{H}\right\}$ NMR (126 MHz, CD ${ }_{3} \mathrm{CN}$ ): $\delta 138.0(\mathrm{CH}), 125.1(\mathrm{CH}), 119.4(\mathrm{CN}), 99.3$ (Cq), $95.9(\mathrm{Cq}), 71.8\left(\mathrm{CH}_{2}\right), 64.5(\mathrm{CH}), 59.8(\mathrm{CH}), 57.8\left(\mathrm{CH}_{3}\right), 38.9(\mathrm{Cq}), 31.3(\mathrm{Cq}), 28.5\left(\mathrm{CH}_{2}\right), 28.5\left(\mathrm{CH}_{3}\right), 26.8$ $\left(\mathrm{CH}_{3}\right) . \mathrm{IR} \vee\left(\mathrm{cm}^{-1}\right)=3031,2972,2222,994,963,846,763,711,679$. HRMS (ESI) : $\mathrm{m} / \mathrm{z}[\mathrm{M}+\mathrm{H}]^{+}$calcd for $\left[\mathrm{C}_{14} \mathrm{H}_{18} \mathrm{NO}_{2}\right]^{+}: 232.1338$, found : 232.1339 .

\section{2a1-methoxy-2a,2a1,2b,2c,3,4,5,6,7,8-decahydro-9-oxabenzo[a]-}

cyclopenta[h]cyclopropa[cd]pentalene-2a-carbonitrile (11d). The product was prepared following the general procedure for photochemical reactions using 4-(2-(cyclohex-1-en-1-yl)ethoxy)-3methoxybenzonitrile $10 \mathrm{~d}(39 \mathrm{mg}, 0.15 \mathrm{mmol})$ and $\mathrm{MeCN}(8.5 \mathrm{~mL})$. The tubes were irradiated at a wavelength of $300 \mathrm{~nm}$ for $2 \mathrm{~h} 30$. Column chromatography $\left(\mathrm{SiO}_{2}\right.$, petroleum ether/ethyl acetate $=9: 1$, $\mathrm{KMnO}_{4}$ ) afforded the product $11 \mathrm{~d}(15.5 \mathrm{mg}, 39 \%)$ as a colorless oil. $R f=0.35$ (petroleum ether/ethyl acetate = 9:1, UV). ${ }^{1} \mathrm{H}$ NMR $\left(500 \mathrm{MHz}, \mathrm{CD}_{3} \mathrm{CN}\right): \delta 5.86(\mathrm{~d}, J=5.9 \mathrm{~Hz}, 1 \mathrm{H} ; \mathrm{CH}), 5.78(\mathrm{~d}, J=5.9 \mathrm{~Hz}, 1 \mathrm{H} ; \mathrm{CH})$, $4.09\left(\mathrm{~m}, 1 \mathrm{H} ; \mathrm{CH}_{2}\right), 3.96\left(\mathrm{~m}, 1 \mathrm{H} ; \mathrm{CH}_{2}\right), 3.62\left(\mathrm{~s}, 3 \mathrm{H} ; \mathrm{CH}_{3}\right), 2.97(\mathrm{~d}, J=6.4 \mathrm{~Hz}, 1 \mathrm{H} ; \mathrm{CH}), 2.38\left(\mathrm{~m}, 1 \mathrm{H}, \mathrm{CH}_{2}\right), 2.27$ $\left(\mathrm{m}, 1 \mathrm{H} ; \mathrm{CH}_{2}\right), 1.76\left(\mathrm{~m}, 1 \mathrm{H} ; \mathrm{CH}_{2}\right), 1.64\left(\mathrm{~m}, 3 \mathrm{H} ; \mathrm{CH}_{2}\right), 1.44\left(\mathrm{~m}, 1 \mathrm{H} ; \mathrm{CH}_{2}\right), 1.35\left(\mathrm{~m}, 1 \mathrm{H} ; \mathrm{CH}_{2}\right), 1.22\left(\mathrm{~m}, 3 \mathrm{H} ; \mathrm{CH}_{2}\right)$. ${ }^{13} \mathrm{C}\left\{{ }^{1} \mathrm{H}\right\}$ NMR $\left(126 \mathrm{MHz}, \mathrm{CD}_{3} \mathrm{CN}\right): \delta 136.9(\mathrm{CH}), 126.0(\mathrm{CH}), 119.6(\mathrm{CN}), 100.1(\mathrm{Cq}), 97.1(\mathrm{Cq}), 69.3\left(\mathrm{CH}_{2}\right), 59.4$ (Cq), $57.6\left(\mathrm{CH}_{3}\right), 54.0(\mathrm{CH}), 41.1\left(\mathrm{CH}_{2}\right), 39.7(\mathrm{CH}), 31.1\left(\mathrm{CH}_{2}\right), 30.1(\mathrm{Cq}), 27.3\left(\mathrm{CH}_{2}\right), 24.0\left(\mathrm{CH}_{2}\right), 22.7\left(\mathrm{CH}_{2}\right) . \mathrm{IR}$ $v\left(\mathrm{~cm}^{-1}\right)=3072,2926,2226,964,836,794,722$. HRMS (ESI) : $\mathrm{m} / z[\mathrm{M}+\mathrm{H}]^{+}$calcd for $\left[\mathrm{C}_{16} \mathrm{H}_{20} \mathrm{NO}_{2}\right]^{+}: 258.1494$ , found : 258.1493 .

\section{3a,4,4-trifluoro-4a1-methoxy-3,3a,4,4a,4a1,4b-hexahydro-2H-cyclo-}

propa[3,4]pentaleno[6a,1-b]furan-4b-carbonitrile (11e). The product was prepared following the general procedure for photochemical reactions using 3-methoxy-4-((3,4,4-trifluorobut-3-en1-yl)oxy)benzonitrile 10e $(68 \mathrm{mg}, 0.26 \mathrm{mmol})$ and $\mathrm{MeCN}(17 \mathrm{~mL})$. The tube was irradiated at a wavelength of $300 \mathrm{~nm}$ for $1 \mathrm{~h} 40$. Column chromatography $\left(\mathrm{SiO}_{2}\right.$, petroleum ether/ethyl acetate =9:1, $\left.\mathrm{KMnO}_{4}\right)$ afforded the product 11 e (13 mg, 19\%) as a colorless oil. Product 11e was crystallized as colorless crystals via slow evaporation of a petroleum ether/diethyl ether (1:1) solution at room temperature. Mp. : $91^{\circ} \mathrm{C} \cdot R f=0.26$ 
(petroleum ether/ethyl acetate = 9:1, UV). ${ }^{1} \mathrm{H} \mathrm{NMR}\left(500 \mathrm{MHz}, \mathrm{CDCl}_{3}\right): \delta 5.97(\mathrm{dd}, J=5.9,1.0 \mathrm{~Hz} 1 \mathrm{H} ; \mathrm{CH})$, $5.86(\mathrm{dd}, J=5.9,1.5 \mathrm{~Hz}, 1 \mathrm{H} ; \mathrm{CH}), 4.41\left(\mathrm{~m}, 1 \mathrm{H} ; \mathrm{CH}_{2}\right), 4.36\left(\mathrm{~m}, 1 \mathrm{H} ; \mathrm{CH}_{2}\right), 3.70\left(\mathrm{~s}, 3 \mathrm{H} ; \mathrm{CH}_{3}\right), 3.15(\mathrm{~m}, 1 \mathrm{H} ; \mathrm{CH})$, $2.68\left(\mathrm{~m}, 2 \mathrm{H} ; \mathrm{CH}_{2}\right), 2.35\left(\mathrm{~m}, 1 \mathrm{H} ; \mathrm{CH}_{2}\right), 2.11\left(\mathrm{~m}, 1 \mathrm{H} ; \mathrm{CH}_{2}\right), 1.89\left(\mathrm{~m}, 1 \mathrm{H} ; \mathrm{CH}_{2}\right), 1.13\left(\mathrm{~s}, 3 \mathrm{H} ; \mathrm{CH}_{3}\right), 1.06(\mathrm{~s}, 3 \mathrm{H}$; $\left.\mathrm{CH}_{3}\right) \cdot{ }^{13} \mathrm{C}\left\{{ }^{1} \mathrm{H}\right\} \operatorname{NMR}\left(126 \mathrm{MHz}, \mathrm{CDCl}_{3}\right): \delta 132.6(\mathrm{CH}), 125.9(\mathrm{CH}), 117.8$ (ddd, $J=264.5,253.8,21.4 \mathrm{~Hz}, \mathrm{CF}_{2}$ ) 115.7 (CN), 111.1 (ddd, $J=221.3,21.0,20.9 \mathrm{~Hz}, \mathrm{CF}$ ), 97.0 (dd, $J=18.2,8.7 \mathrm{~Hz}, \mathrm{Cq}$ ), 87.8 (virt. t, $J=9.2 \mathrm{~Hz}$, Cq), $70.3\left(\mathrm{CH}_{2}\right), 58.8\left(\mathrm{CH}_{3}\right), 46.3$ (ddd, $J=38.0,25.3,3.2 \mathrm{~Hz}, \mathrm{CH}$ ), 30.7 (virt. dd, $J=22.8,3.8 \mathrm{~Hz}, \mathrm{CH}_{2}$ ), 29.9 (m, Cq). ${ }^{19} \mathrm{~F} \mathrm{NMR}\left(471 \mathrm{MHz}, \mathrm{CDCl}_{3}\right): \delta-93.3$ (virt. ddt, $\left.J=251.2,11.0,2.1 \mathrm{~Hz}, 1 \mathrm{~F} ; \mathrm{C}-\mathrm{F}\right),-107.6$ (virt. dd, $J=$ 251.2, $5.1 \mathrm{~Hz}, 1 \mathrm{~F} ; \mathrm{C}-\mathrm{F}_{2}$ ), -171.6 (virt. ddtd, $\left.J=35.7,19.6,4.8,2.7 \mathrm{~Hz}, 1 \mathrm{~F} ; \mathrm{C}-\mathrm{F}_{2}\right) . \mathrm{IR} v\left(\mathrm{~cm}^{-1}\right)=3068,2912,2240$, 992, 945, 914, 831, 758, 683.

HRMS (ESI) : $m / z[M+H]^{+}$calcd for $\left[\mathrm{C}_{12} \mathrm{H}_{11} \mathrm{NO}_{2} \mathrm{~F}_{3}\right]^{+}: 258.0742$, found : 258.0742 .

\section{4a1,6-dimethoxy-3a-methyl-3,3a,4,4a,4a1,4b-hexahydro-2H-cyclo-}

propa[3,4]pentaleno[6a,1-b]furan-4b-carbonitrile (11f). The product was prepared following the general procedure for photochemical reactions using 4-(but-3-en-1-yloxy)-3,5dimethoxybenzonitrile $10 \mathrm{f}(62 \mathrm{mg}, 0.27 \mathrm{mmol})$ and $\mathrm{MeCN}(15 \mathrm{~mL})$. The tube was irradiated at a wavelength of $300 \mathrm{~nm}$ for $1 \mathrm{~h} 30$. Column chromatography $\left(\mathrm{SiO}_{2}\right.$, petroleum ether/ethyl acetate $\left.=9: 1, \mathrm{KMnO}_{4}\right)$ afforded the product $11 \mathrm{f}(39 \mathrm{mg}, 63 \%$ ) as a colorless oil. $R f=0.14$ (petroleum ether/ethyl acetate $=9: 1, \mathrm{UV}) .{ }^{1} \mathrm{H}$ NMR (500 MHz, CDCl $): \delta 4.50(\mathrm{~s}, 1 \mathrm{H}, \mathrm{CH}), 4.30\left(\mathrm{~m}, 1 \mathrm{H} ; \mathrm{CH}_{2}\right), 4.05\left(\mathrm{~m}, 1 \mathrm{H} ; \mathrm{CH}_{2}\right), 3.70\left(\mathrm{~s}, 3 \mathrm{H} ; \mathrm{CH}_{3}\right), 3.68(\mathrm{~s}$, $\left.3 \mathrm{H} ; \mathrm{CH}_{3}\right), 2.64(\mathrm{~d}, J=6.3 \mathrm{~Hz}, 1 \mathrm{H} ; \mathrm{CH}), 2.56(\mathrm{ddd}, J=11.4,7.5,6.4 \mathrm{~Hz}, 1 \mathrm{H} ; \mathrm{CH}), 2.09-1.97\left(\mathrm{~m}, 1 \mathrm{H} ; \mathrm{CH}_{2}\right), 1.95$ (dd, $J=14.7,6.3 \mathrm{~Hz}, 1 \mathrm{H} ; \mathrm{CH}), 1.62$ (dd, $\left.J=14.7,6.4 \mathrm{~Hz}, 1 \mathrm{H} ; \mathrm{CH}_{2}\right) \cdot{ }^{13} \mathrm{C}\left\{{ }^{1} \mathrm{H}\right\} \mathrm{NMR}\left(126 \mathrm{MHz}, \mathrm{CDCl}_{3}\right): \delta 160.9$ $(\mathrm{Cq}), 119.1(\mathrm{Cq}), 94.3(\mathrm{Cq}), 87.5(\mathrm{Cq}), 86.8(\mathrm{CH}), 72.0\left(\mathrm{CH}_{2}\right), 57.7\left(\mathrm{CH}_{3}\right), 57.4\left(\mathrm{CH}_{3}\right), 54.1(\mathrm{CH}), 44.6(\mathrm{CH}), 29.4$ $\left(\mathrm{CH}_{2}\right), 24.9(\mathrm{Cq}), 23.8\left(\mathrm{CH}_{2}\right) . \mathrm{IR} \vee\left(\mathrm{cm}^{-1}\right)=2941,2223,1644,1620,945,874,732,702,687 . \mathrm{HRMS}(\mathrm{ESI}): \mathrm{m} / \mathrm{z}$ $[\mathrm{M}+\mathrm{H}]^{+}$calcd for $\left[\mathrm{C}_{13} \mathrm{H}_{16} \mathrm{NO}_{3}\right]^{+}: 234.1130$, found : 234.1129 .

\section{4a1-methoxy-3,3a,4,4a,4a1,6a-hexahydro-2H-cyclopropa[1,6]-penta-leno[1,2-}

b]furan-5-carbonitrile (12a). The product was prepared following the general procedure for photochemical reactions using 4-(but-3-en-1-yloxy)-3-methoxybenzonitrile 10a (54 mg, $0.27 \mathrm{mmol}$ ) and MeCN (15 mL). The tube was irradiated at a wavelength of $300 \mathrm{~nm}$ for $2 \mathrm{~h} 30$. Column chromatography $\left(\mathrm{SiO}_{2}\right.$, petroleum ether/ethyl acetate $\left.=9: 1, \mathrm{KMnO}_{4} / \mathrm{UV}\right)$ afforded the products $12 \mathrm{a}(17 \mathrm{mg}, 31 \%)$ as a colorless oil. $R f=0.1$ (petroleum ether/ethyl acetate $=9: 1, \mathrm{UV}) \cdot{ }^{1} \mathrm{H} \mathrm{NMR}\left(500 \mathrm{MHz}, \mathrm{CDCl}_{3}\right): \delta 6.64(\mathrm{~d}, J=$ $3.0 \mathrm{~Hz}, 1 \mathrm{H} ; \mathrm{CH}$ ), 4.22 (ddd, J=8.6, 7.0, $4.4 \mathrm{~Hz}, 1 \mathrm{H} ; \mathrm{CH}_{2}$ ), 3.97 (ddd, J=9.0,8.6, 7.0 Hz, $\left.1 \mathrm{H} ; \mathrm{CH}_{2}\right), 3.58(\mathrm{~d}, J=$ $5.4 \mathrm{~Hz}, 1 \mathrm{H} ; \mathrm{CH}$ ), $3.45\left(\mathrm{~s}, 3 \mathrm{H} ; \mathrm{CH}_{3}\right), 2.66(\mathrm{~d}, J=3.0 \mathrm{~Hz}, 1 \mathrm{H} ; \mathrm{CH}), 2.48$ (dddd, J=10.0, 9.4, 6.5, $4.1 \mathrm{~Hz}, 1 \mathrm{H} ; \mathrm{CH}$ ), 2.19 (dddd, $J=12.5,10.0,9.0,7.3 \mathrm{~Hz}, 1 \mathrm{H} ; \mathrm{CH}_{2}$ ), 2.16 (dd, $J=12.4,6.5 \mathrm{~Hz}, 1 \mathrm{H} ; \mathrm{CH}_{2}$ ) 1.97 (ddd, $J=12.4,9.4$, 
$5.4 \mathrm{~Hz}, 1 \mathrm{H} ; \mathrm{CH}_{2}$ ), 1.74 (dddd, $\left.J=12.5,7.0,4.4,4.1 \mathrm{~Hz}, 1 \mathrm{H} ; \mathrm{CH}_{2}\right) .{ }^{13} \mathrm{C}\left\{{ }^{1} \mathrm{H}\right\} \mathrm{NMR}\left(126 \mathrm{MHz}, \mathrm{CDCl}_{3}\right): \delta 146.9$ $(\mathrm{CH}), 116.1(\mathrm{Cq}), 116.0(\mathrm{Cq}), 86.4(2 \mathrm{Cq}), 71.9\left(\mathrm{CH}_{2}\right), 58.2\left(\mathrm{CH}_{3}\right), 55.3(\mathrm{CH}), 42.7\left(\mathrm{CH}_{2}\right), 39.6(\mathrm{CH}), 39.2(\mathrm{CH})$, $32.2\left(\mathrm{CH}_{2}\right) . \mathrm{IR} \vee\left(\mathrm{cm}^{-1}\right)=2944,2213,1618,1586,968,895,872,680$. HRMS (ESI) : $\mathrm{m} / \mathrm{z}[\mathrm{M}+\mathrm{H}]^{+}$calcd for $\left[\mathrm{C}_{12} \mathrm{H}_{14} \mathrm{NO}_{2}\right]^{+}:$204.1025, found : 204.1023.

\section{2a1-methoxy-2a,2a1,2b,3,4,5,6,7,8,9b-decahydro-9-oxabenzo[a]- cyclopenta[b]cyclopropa[cd]pentalene-2-carbonitrile (12d).}

The product was prepared following the general procedure for photochemical reactions using 4-(2(cyclohex-1-en-1-yl)ethoxy)-3-methoxybenzonitrile $10 \mathrm{~d}(52 \mathrm{mg}, 0.2 \mathrm{mmol})$ and $\mathrm{MeCN}(110 \mathrm{~mL})$ distributed in 6 quartz glass tubes. The tubes were irradiated at a wavelength of $300 \mathrm{~nm}$ for 25 minutes. Column chromatography $\left(\mathrm{SiO}_{2}\right.$, petroleum ether/ethyl acetate $\left.=9: 1, \mathrm{KMnO}_{4} / \mathrm{UV}\right)$ afforded the product $\mathbf{1 2 d}(9 \mathrm{mg}$, $17 \%$ ) as a colorless oil. $R f=0.22$ (petroleum ether/ethyl acetate $=9: 1, \mathrm{UV}) .{ }^{1} \mathrm{H}$ NMR $\left(500 \mathrm{MHz}, \mathrm{CD}_{3} \mathrm{CN}\right): \delta$ $6.82\left(\mathrm{~d}, J=3.2 \mathrm{~Hz}, 1 \mathrm{H} ; \mathrm{CH}\right.$ ), 4.10 (ddd, $J=8.6,7.3,6.0 \mathrm{~Hz}, 1 \mathrm{H} ; \mathrm{CH}_{2}$ ), 3.83 (ddd, J = 8.6, 7.8, $6.8 \mathrm{~Hz}, 1 \mathrm{H} ; \mathrm{CH}_{2}$ ), $3.51(\mathrm{~d}, J=5.9 \mathrm{~Hz}, 1 \mathrm{H} ; \mathrm{CH}), 3.37\left(\mathrm{~s}, 3 \mathrm{H} ; \mathrm{CH}_{3}\right), 2.56$ (ddd, J = 8.3, 5.9, $\left.2.6 \mathrm{~Hz}, 1 \mathrm{H} ; \mathrm{CH}\right), 2.50$ (d, J = $3.2 \mathrm{~Hz}, 1 \mathrm{H}$; $\mathrm{CH}), 2.00-1.84\left(\mathrm{~m}, 2 \mathrm{H} ; \mathrm{CH}_{2}\right), 1.81-1.74\left(\mathrm{~m}, 1 \mathrm{H} ; \mathrm{CH}_{2}\right), 1.73-1.66\left(\mathrm{~m}, 1 \mathrm{H} ; \mathrm{CH}_{2}\right), 1.66-1.59(\mathrm{~m}, 1 \mathrm{H}), 1.52$ (dt, $J=13.7,4.2 \mathrm{~Hz}, 2 \mathrm{H} ; \mathrm{CH}_{2}$ ), 1.40 (ddq, $J=17.8,13.0,4.4 \mathrm{~Hz}, 2 \mathrm{H} ; \mathrm{CH}_{2}$ ), 1.21 (tdt, J=13.1, 11.3, 3.9 Hz, $1 \mathrm{H}$; $\left.\mathrm{CH}_{2}\right) \cdot{ }^{13} \mathrm{C}\left\{{ }^{1} \mathrm{H}\right\}$ NMR $\left(126 \mathrm{MHz}, \mathrm{CD}_{3} \mathrm{CN}\right): \delta 151.1(\mathrm{CH}), 116.8(\mathrm{CN}), 91.0(\mathrm{Cq}), 90.8(\mathrm{Cq}), 70.2\left(\mathrm{CH}_{2}\right), 62.1(\mathrm{CH})$, $57.8\left(\mathrm{CH}_{3}\right), 55.4(\mathrm{CH}), 50.9(\mathrm{Cq}), 44.0(\mathrm{CH}), 39.0\left(\mathrm{CH}_{2}\right), 33.9\left(\mathrm{CH}_{2}\right), 25.4\left(\mathrm{CH}_{2}\right), 23.1\left(\mathrm{CH}_{2}\right), 22.4\left(\mathrm{CH}_{2}\right) . \mathrm{IR} \mathrm{V}$ $\left(\mathrm{cm}^{-1}\right)=2933,2209,1583,938,925,891,867,803,718$. HRMS (ESI) : $\mathrm{m} / \mathrm{z}[\mathrm{M}+\mathrm{H}]^{+}$calcd for $\left[\mathrm{C}_{16} \mathrm{H}_{20} \mathrm{NO}_{2}\right]^{+}$: 258.1494 , found : 258.1493 .

\section{3a,4,4-trifluoro-4a1-methoxy-3,3a,4,4a,4a1,6a-hexahydro-2H-cyclo-}

propa[1,6]pentaleno[1,2-b]furan-5-carbonitrile (12e). The product was prepared following the general procedure for photochemical reactions using 3-methoxy-4-((3,4,4-trifluorobut-3-en-1yl)oxy)benzonitrile $10 \mathrm{e}(68 \mathrm{mg}, 0.26 \mathrm{mmol})$ and $\mathrm{MeCN}(17 \mathrm{~mL})$. The tube was irradiated at a wavelength of $300 \mathrm{~nm}$ for $1 \mathrm{~h} 40$. Column chromatography $\left(\mathrm{SiO}_{2}\right.$, petroleum ether/ethyl acetate $\left.=9: 1, \mathrm{KMnO}_{4}\right)$ afforded the product $12 \mathrm{e}(29 \mathrm{mg}, 43 \%)$ as a colorless oil. $R f=0.15$ (petroleum ether/ethyl acetate $=9: 1, \mathrm{UV}) .{ }^{1} \mathrm{H}$ NMR (500 MHz, CD 3 CN): $\delta 7.04(\mathrm{td}, J=3.1,1.3 \mathrm{~Hz}, 1 \mathrm{H} ; \mathrm{CH}), 4.32\left(\mathrm{td}, J=8.8,2.4 \mathrm{~Hz}, 1 \mathrm{H} ; \mathrm{CH}_{2}\right), 4.21(\mathrm{~d}, J=$ $10.6 \mathrm{~Hz}, 1 \mathrm{H} ; \mathrm{CH}), 3.68\left(\mathrm{~m}, 1 \mathrm{H} ; \mathrm{CH}_{2}\right), 3.43\left(\mathrm{~s}, 3 \mathrm{H} ; \mathrm{CH}_{3}\right), 2.98(\mathrm{dd}, J=3.1,2.1 \mathrm{~Hz}, 1 \mathrm{H} ; \mathrm{CH}), 2.91-2.79(\mathrm{~m}, 1 \mathrm{H}$; $\mathrm{CH}_{2}$ ), 2.28 (ddddd, $\left.J=30.1,14.1,10.6,8.7,5.6 \mathrm{~Hz}, 1 \mathrm{H} ; \mathrm{CH}_{2}\right) .{ }^{13} \mathrm{C}\left\{{ }^{1} \mathrm{H}\right\} \mathrm{NMR}\left(126 \mathrm{MHz}, \mathrm{CD}_{3} \mathrm{CN}\right): \delta 152.5(\mathrm{CH})$, 125.44 (ddd, $J=276.6,258.5,29.0$ Hz, CF $), 115.7$ (CN), 111.10 (d, $J=9.6 \mathrm{~Hz}, \mathrm{Cq}$ ), 106.4 (ddd, $J=212.4$, 31.1, 23.2 Hz, CF), 84.9 (t, $J=11.1 \mathrm{~Hz}, \mathrm{Cq}$ ), 83.7 (dd, $J=22.3,6.1 \mathrm{~Hz}, \mathrm{Cq}$ ), $70.3\left(\mathrm{~d}, J=2.1 \mathrm{~Hz}, \mathrm{CH}_{2}\right), 60.5$ (dd, $J=29.9,19.5 \mathrm{~Hz}, \mathrm{CH}), 59.2\left(\mathrm{CH}_{3}\right), 38.5(\mathrm{CH}), 34.1\left(\mathrm{dd}, J=25.1,5.7 \mathrm{~Hz}, \mathrm{CH}_{2}\right) .{ }^{19} \mathrm{~F} \mathrm{NMR}\left(471 \mathrm{MHz}, \mathrm{CD}_{3} \mathrm{CN}\right): \delta$ 
$-105.25(\mathrm{dtt}, J=232.5,9.9,4.2 \mathrm{~Hz}$ ), $-116.02(\mathrm{ddd}, J=232.3,10.6,2.0 \mathrm{~Hz}),-155.21$ (dtd, $J=29.5,10.6,8.1$ $\mathrm{Hz}) . \mathrm{IR} \vee\left(\mathrm{cm}^{-1}\right)=3077,2973,2224,1665,1595,985,903,828,711,683$. HRMS (ESI) : $\mathrm{m} / \mathrm{z}[\mathrm{M}+\mathrm{H}]^{+}$calcd for $\left[\mathrm{C}_{12} \mathrm{H}_{11} \mathrm{NO}_{2} \mathrm{~F}_{3}\right]^{+}: 258.0742$, found : 258.0744 .

\section{5-methoxy-3,3a,4,6a-tetrahydro-2H-cyclobuta[h]benzofuran-6a-carbonitrile (13a).}

The product was prepared following the general procedure for photochemical reactions using 4-(but-3en-1-yloxy)-3-methoxybenzonitrile 10a (54 mg, $0.27 \mathrm{mmol}$ ) and $\mathrm{MeCN}(15 \mathrm{~mL})$. The tube was irradiated at a wavelength of $300 \mathrm{~nm}$ for $2 \mathrm{~h} 30$. Column chromatography $\left(\mathrm{SiO}_{2}\right.$, petroleum ether/ethyl acetate $=9: 1$, $\left.\mathrm{KMnO}_{4} / \mathrm{UV}\right)$ afforded an inseparable mixture of the products $13 \mathrm{a}(5 \mathrm{mg}, 9 \%)$ and $12 \mathrm{a}(1.5 \mathrm{mg}, 3 \%)$ as a colorless oil. Analytical data for the product 13a are reported for a mixture with 12a. $R f=0.1$ (petroleum ether/ethyl acetate = 9:1, UV). ${ }^{1} \mathrm{H}$ NMR $\left(500 \mathrm{MHz}, \mathrm{CD}_{3} \mathrm{CN}\right): \delta 6.70(\mathrm{~d}, J=3.0 \mathrm{~Hz}, 1 \mathrm{H} ; \mathrm{CH}, 12 \mathrm{a}), 6.23(\mathrm{~d}, J=$ 2.7 Hz, 1H; CH, 13a), 6.19 (d, J = 2.7 Hz, 1H; 13a), 4.69 (d, J = $1.9 \mathrm{~Hz}, 1 \mathrm{H} ; \mathrm{CH}, 13 \mathrm{a}$ ), 4.12 (ddd, J = 8.7, 7.5, $4.1 \mathrm{~Hz}, 1 \mathrm{H} ; \mathrm{CH}_{2}, 12 \mathrm{a}$ ), 3.98 (td, $J=8.7,3.1 \mathrm{~Hz}, 1 \mathrm{H} ; \mathrm{CH}_{2}, 13 \mathrm{a}$ ), 3.89 (td, $J=8.8,7.2 \mathrm{~Hz}, 1 \mathrm{H} ; \mathrm{CH}_{2}, 13 \mathrm{a}$ ), 3.84 (td, $\left.J=8.7,6.9 \mathrm{~Hz}, 1 \mathrm{H} ; \mathrm{CH}_{2}, 12 \mathrm{a}\right), 3.59(\mathrm{~d}, J=5.4 \mathrm{~Hz}, 1 \mathrm{H} ; \mathrm{CH}, 12 \mathrm{a}), 3.52\left(\mathrm{~s}, 3 \mathrm{H} ; \mathrm{CH}_{3}, 13 \mathrm{a}\right), 3.39\left(\mathrm{~s}, 3 \mathrm{H} ; \mathrm{CH}_{3}, 12 \mathrm{a}\right)$, 2.60 (d, $J=2.9 \mathrm{~Hz}, 1 \mathrm{H} ; \mathrm{CH}, 12 \mathrm{a}$ ), 2.47 (dddd, $J=10.7,7.3,5.7,3.0 \mathrm{~Hz}, 1 \mathrm{H} ; \mathrm{CH}, 13 \mathrm{a}), 2.43-2.35$ (m, 1H; CH, 12a), 2.29 (ddd, $J=16.9,5.7,2.0 \mathrm{~Hz}, 1 \mathrm{H} ; \mathrm{CH}_{2}, 13 \mathrm{a}$ ), 2.22 (dd, J = 16.9, 3.1 Hz, 1H; $\mathrm{CH}_{2}, 13 \mathrm{a}$ ), 2.21 - 2.17 (m, $\left.1 \mathrm{H} ; \mathrm{CH}_{2}, 12 \mathrm{a}\right), 2.12-2.07\left(\mathrm{~m}, 1 \mathrm{H} ; \mathrm{CH}_{2}, 12 \mathrm{a}\right), 2.07-2.04\left(\mathrm{~m}, 1 \mathrm{H} ; \mathrm{CH}_{2}, 12 \mathrm{a}\right), 2.04-2.01\left(\mathrm{~m}, 1 \mathrm{H} ; \mathrm{CH}_{2}, 13 \mathrm{a}\right)$, 1.87 (ddt, $J=12.2,11.0,9.2 \mathrm{~Hz}, 1 \mathrm{H}, 13 \mathrm{a}), 1.69$ (ddt, $\left.J=12.5,7.6,4.0 \mathrm{~Hz}, 1 \mathrm{H} ; \mathrm{CH}_{2}, 12 \mathrm{a}\right) .{ }^{13} \mathrm{C}\left\{{ }^{1} \mathrm{H}\right\} \mathrm{NMR}(126$ $\mathrm{MHz}, \mathrm{CDCl}_{3}$ ): $\delta 157.7$ (Cq, 13a), 148.3 (CH, 12a), 140.0 (CH, 13a), 138.4 (CH, 13a), 122.1 (CN, 13a), 90.9 (CH, 13a), 89.7 (Cq, 13a), 87.1 (Cq, 12a), 72.1 (Cq, 13a), $67.6\left(\mathrm{CH}_{2}, 13 a\right), 58.0\left(\mathrm{CH}_{3}, 12 \mathrm{a}\right), 56.2(\mathrm{CH}, 12 \mathrm{a}) 55.4$ $\left(\mathrm{CH}_{3}, 13 \mathrm{a}\right), 50.7(\mathrm{CH}, 12 \mathrm{a}), 43.0\left(\mathrm{CH}_{2}, 12 \mathrm{a}\right), 40.2(\mathrm{CH}, 12 \mathrm{a}), 40.0(\mathrm{CH}, 12 \mathrm{a}), 39.5(\mathrm{CH}, 13 \mathrm{a}), 32.6\left(\mathrm{CH}_{2}, 12 \mathrm{a}\right)$, $28.7\left(\mathrm{CH}_{2}, 13 a\right), 28.4\left(\mathrm{CH}_{2}, 13 a\right)$. IR $\vee\left(\mathrm{cm}^{-1}\right)=2940,2216,867,680$. HRMS (ESI) : $\mathrm{m} / z[\mathrm{M}+\mathrm{H}]^{+}$calcd for $\left[\mathrm{C}_{12} \mathrm{H}_{14} \mathrm{NO}_{2}\right]^{+}:$204.1025, found : 204.1024.

\section{5-methoxy-3a-methyl-3,3a,4,6a-tetrahydro-2H-cyclobuta[h]-benzofuran-6a-}

carbonitrile (13b). The product was prepared following the general procedure for photochemical reactions using 3-methoxy-4-((3-methylbut-3-en-1-yl)oxy)benzonitrile 10b (58 mg, $0.27 \mathrm{mmol}$ ) and MeCN $(15 \mathrm{~mL})$. The tube was irradiated at a wavelength of $300 \mathrm{~nm}$ for $2 \mathrm{~h} 30$. Column chromatography $\left(\mathrm{SiO}_{2}\right.$, petroleum ether/ethyl acetate $\left.=9: 1, \mathrm{KMnO}_{4} / \mathrm{UV}\right)$ afforded the product $\mathbf{1 3 b}(6 \mathrm{mg}, 10 \%)$ as a colorless oil. $R f=0.1$ (petroleum ether/ethyl acetate $=9: 1, \mathrm{UV}) .{ }^{1} \mathrm{H}$ NMR $\left(500 \mathrm{MHz}, \mathrm{CD}_{3} \mathrm{CN}\right): \delta 6.26(\mathrm{~d}, J=2.8 \mathrm{~Hz}, 1 \mathrm{H}$; $\mathrm{CH}), 6.23(\mathrm{~d}, \mathrm{~J}=2.8 \mathrm{~Hz}, 1 \mathrm{H} ; \mathrm{CH}), 4.69(\mathrm{~d}, \mathrm{~J}=2.1 \mathrm{~Hz}, 1 \mathrm{H} ; \mathrm{CH}), 4.02-3.90\left(\mathrm{~m}, 2 \mathrm{H} ; \mathrm{CH}_{2}\right), 3.52\left(\mathrm{~s}, 3 \mathrm{H} ; \mathrm{CH}_{3}\right), 2.19$ - $2.16\left(\mathrm{~m}, 1 \mathrm{H} ; \mathrm{CH}_{2}\right), 2.12-2.05\left(\mathrm{~m}, 2 \mathrm{H} ; \mathrm{CH}_{2}\right), 1.71$ (ddd, $\left.J=12.4,6.8,3.7 \mathrm{~Hz}, 1 \mathrm{H} ; \mathrm{CH}_{2}\right), 1.06(\mathrm{~d}, J=0.7 \mathrm{~Hz}$, 3H; $\left.\mathrm{CH}_{3}\right) .{ }^{13} \mathrm{C}\left\{{ }^{1} \mathrm{H}\right\}$ NMR (126 MHz, CD $\left.{ }_{3} \mathrm{CN}\right): \delta 157.9(\mathrm{Cq}), 139.2(\mathrm{CH}), 138.5(\mathrm{CH}), 122.1(\mathrm{CN}), 92.2(\mathrm{Cq}), 90.7$ 
$(\mathrm{CH}), 66.0\left(\mathrm{CH}_{2}\right), 55.4\left(\mathrm{CH}_{3}\right), 51.3(\mathrm{Cq}), 43.1(\mathrm{Cq}), 36.3\left(\mathrm{CH}_{2}\right), 34.5\left(\mathrm{CH}_{2}\right), 23.1\left(\mathrm{CH}_{3}\right) . \mathrm{IR} v\left(\mathrm{~cm}^{-1}\right)=2925,2230$, $1661,979,888,800,780,709$. HRMS (ESI) : $m / z[M+H]^{+}$calcd for $\left[\mathrm{C}_{13} \mathrm{H}_{16} \mathrm{NO}_{2}\right]^{+}: 218.1181$, found : 218.1181.

\section{5-methoxy-4,4-dimethyl-3,3a,4,6a-tetrahydro-2H-cyclobuta[h]benzo-furan-6a-}

carbonitrile $(\mathbf{1 3 c})$. The product was prepared following the general procedure for photochemical reactions using 3-methoxy-4-((4-methylpent-3-en-1-yl)oxy)benzonitrile 10c (61.7 mg, $0.27 \mathrm{mmol})$ and $\mathrm{MeCN}(15 \mathrm{~mL})$. The tube was irradiated at a wavelength of $300 \mathrm{~nm}$ for $2 \mathrm{~h}$. Column chromatography $\left(\mathrm{SiO}_{2}\right.$, petroleum ether/ethyl acetate $\left.=9: 1, \mathrm{KMnO}_{4}\right)$ afforded the product $13 \mathrm{c}(8.6 \mathrm{mg}, 14 \%)$ as a colorless oil. Product $13 \mathrm{c}$ was crystallized as colorless crystals via slow evaporation of a petroleum ether/diethyl ether (1:1) solution at room temperature. Mp. : $116{ }^{\circ} \mathrm{C} . R f=0.33$ (petroleum ether/ethyl acetate $=9: 1, \mathrm{UV}$ ). ${ }^{1} \mathrm{H}$ NMR (500 MHz, CD ${ }_{3} \mathrm{CN}$ ): $\delta 6.32(\mathrm{~d}, J=2.7 \mathrm{~Hz}, 1 \mathrm{H} ; \mathrm{CH}), 6.30$ (d, J = 2.7 Hz, 1H; CH), $4.67(\mathrm{~s}, 1 \mathrm{H} ; \mathrm{CH}), 4.00$ (ddd, J = 9.4, 8.2, $\left.2.4 \mathrm{~Hz}, 1 \mathrm{H} ; \mathrm{CH}_{2}\right), 3.90-3.80\left(\mathrm{~m}, 2 \mathrm{H} ; \mathrm{CH}, \mathrm{CH}_{2}\right), 3.54\left(\mathrm{~s}, 3 \mathrm{H} ; \mathrm{CH}_{3}\right), 2.26(\mathrm{dd}, J=11.6,7.0 \mathrm{~Hz}$, $1 \mathrm{H} ; \mathrm{CH}), 2.17-2.08\left(\mathrm{~m}, 1 \mathrm{H} ; \mathrm{CH}_{2}\right), 1.99-1.84\left(\mathrm{~m}, 1 \mathrm{H} ; \mathrm{CH}_{2}\right), 1.14\left(\mathrm{~s}, 3 \mathrm{H} ; \mathrm{CH}_{3}\right), 1.09\left(\mathrm{~s}, 3 \mathrm{H} ; \mathrm{CH}_{3}\right) .{ }^{13} \mathrm{C}\left\{{ }^{1} \mathrm{H}\right\} \mathrm{NMR}$ (126 MHz, $\mathrm{CDCl}_{3}$ ): $\delta 163.1(\mathrm{Cq}), 142.1(\mathrm{CH}), 140.5(\mathrm{CH}), 121.8(\mathrm{CN}), 90.0(\mathrm{CH}), 88.2(\mathrm{Cq}), 66.5\left(\mathrm{CH}_{2}\right), 55.6$ $(\mathrm{CH}), 51.4(\mathrm{CH}), 50.6(\mathrm{Cq}), 37.8(\mathrm{Cq}), 28.4\left(\mathrm{CH}_{2}\right), 27.4\left(\mathrm{CH}_{3}\right), 25.6\left(\mathrm{CH}_{3}\right) . \mathrm{IR} \vee\left(\mathrm{cm}^{-1}\right)=2989,2971,2230,1644$, $986,934,805,788,769,678$. HRMS (ESI) : $m / z[\mathrm{M}+\mathrm{H}]^{+}$calcd for $\left[\mathrm{C}_{14} \mathrm{H}_{18} \mathrm{NO}_{2}\right]^{+}: 232.1338$, found : 232.1338 .

\section{4a-methoxy-4,4-dimethyl-2,3,3a,4,4a,6a-hexahydrocyclobuta-[f]benzo-furan-6-}

carbonitrile (14c). The product was prepared following the general procedure for photochemical reactions using 3-methoxy-4-((4-methylpent-3-en-1-yl)oxy)benzonitrile 10c (61.7 mg, $0.27 \mathrm{mmol})$ and MeCN (15 mL). The tube was irradiated at a wavelength of $300 \mathrm{~nm}$ for $2 \mathrm{~h}$. Column chromatography $\left(\mathrm{SiO}_{2}\right.$, petroleum ether/ethyl acetate $=9: 1, \mathrm{KMnO}_{4}$ ) afforded a $7: 3$ mixture of diastereomers $14 \mathrm{c}$ and $\mathbf{1 4 c ^ { \prime }}$ (16 $\mathrm{mg}, 26 \%)$ as a colorless oil. The stereochemistry of the syn diastereomer (14c, major) and anti diastereomer (14c', minor) were resolved by 2D NMR NOESY experiment (cf ESI S32). Analytical data are reported for the mixture of diastereomers $\mathbf{1 4 c}$ (major) and $\mathbf{1 4 c ^ { \prime }}$ (minor). $R f=0.30$ (petroleum ether/ethyl acetate = 9:1, UV). ${ }^{1} \mathrm{H} \mathrm{NMR}\left(600 \mathrm{MHz}, \mathrm{CDCl}_{3}\right): \delta 6.85$ (d, $J=2.2 \mathrm{~Hz}, 1 \mathrm{H} ; \mathrm{CH}$, major), $6.81(\mathrm{~d}, J=0.7 \mathrm{~Hz}, 1 \mathrm{H}$; $\mathrm{CH}$, minor), 4.82 (dd, $J=6.2,2.6 \mathrm{~Hz}, 1 \mathrm{H} ; \mathrm{CH}, \operatorname{minor}), 4.78$ (td, $J=2.7,0.7 \mathrm{~Hz}, 1 \mathrm{H} ; \mathrm{CH}$, major), 4.24 (td, $J=$ 8.5, $1.5 \mathrm{~Hz}, 1 \mathrm{H} ; \mathrm{CH}_{2}$, major), $4.24\left(\mathrm{~m}, 1 \mathrm{H} ; \mathrm{CH}_{2}\right.$, minor), $4.06-3.96\left(\mathrm{~m}, 1 \mathrm{H} ; \mathrm{CH}_{2}\right.$, major), $3.99-3.93(\mathrm{~m}, 1 \mathrm{H}$; $\mathrm{CH}_{2}$, minor), 3.85 (q, $J=2.6 \mathrm{~Hz}, 1 \mathrm{H} ; \mathrm{CH}$, major), 3.68 (dd, $J=6.3,0.7 \mathrm{~Hz}, 1 \mathrm{H} ; \mathrm{CH}$, minor), $3.34\left(\mathrm{~s}, 3 \mathrm{H} ; \mathrm{CH}_{3}\right.$, major), 3.32 (s, 3H; $\mathrm{CH}_{3}$, minor), 2.84 (ddt, $J=11.2,8.6,2.6 \mathrm{~Hz}, 1 \mathrm{H} ; \mathrm{CH}$, major), 2.42 (ddd, $J=11.0,8.7,2.6$ $\mathrm{Hz}, 1 \mathrm{H} ; \mathrm{CH}$, minor), $2.04-1.97\left(\mathrm{~m}, 1 \mathrm{H} ; \mathrm{CH}_{2}\right.$, minor), $1.97-1.92\left(\mathrm{~m}, 1 \mathrm{H} ; \mathrm{CH}_{2}\right.$, major), $1.92-1.88(\mathrm{~m}, 1 \mathrm{H}$; $\mathrm{CH}_{2}$, minor ), 1.77 (dtd, $\mathrm{J}=12.2,11.1,8.6 \mathrm{~Hz}, 1 \mathrm{H} ; \mathrm{CH}_{2}$, major), 1.09 (s, 3H; $\mathrm{CH}_{3}$, major), 1.04 (s, 3H; $\mathrm{CH}_{3}$, minor), 0.84 (s, 3H; $\mathrm{CH}_{3}$, minor), 0.72 (s, 3H; $\mathrm{CH}_{3}$, major). ${ }^{13} \mathrm{C}\left\{{ }^{1} \mathrm{H}\right\} \mathrm{NMR}$ (151 MHz, $\mathrm{CDCl}_{3}$ ): $\delta 161.1$ (Cq, major), 
160.4 (Cq, minor), 152.5 (CH, major), 149.6 (CH, minor), 121.3 (Cq, major), 120.5 (Cq, minor), 113.4 (Cq, minor), 113.3 (Cq, major), 90.1 ( $\mathrm{Cq}$, minor), 87.3 (Cq, major), 86.3 (CH, minor), 86.0 ( $\mathrm{CH}$, major), $71.0\left(\mathrm{CH}_{2}\right.$, major), $70.6\left(\mathrm{CH}_{2}\right.$, minor), $52.4\left(\mathrm{CH}_{3}\right.$, minor), $52.1\left(\mathrm{CH}_{3}\right.$, major), $48.6(\mathrm{CH}$, major), 47.9 ( $\mathrm{CH}$, minor), 45.4 ( $\mathrm{CH}$, minor), 45.0 ( $\mathrm{CH}$, major), 39.9 ( $\mathrm{Cq}$, major), 39.8 ( $\mathrm{Cq}$, minor), $25.1\left(\mathrm{CH}_{2}\right.$, minor), $25.1\left(\mathrm{CH}_{2}\right.$, major), 22.8 ( $\mathrm{CH}_{3}$, major), $22.4\left(\mathrm{CH}_{3}\right.$, minor), $21.4\left(\mathrm{CH}_{3}\right.$, major), $14.2\left(\mathrm{CH}_{3}\right.$, minor). IR $v\left(\mathrm{~cm}^{-1}\right)=2972,2950,2218,1679$, $989,942,876,800,789,711$. HRMS (ESI) : $m / z[\mathrm{M}+\mathrm{H}]^{+}$calcd for $\left[\mathrm{C}_{14} \mathrm{H}_{18} \mathrm{NO}_{2}\right]^{+}: 232.1338$, found : 232.1339.

\section{0a-methoxy-2,3,4,5,6,8a,10a,10b-octahydro-1H-cyclo-buta[3,4]-naphtho[1,8a-}

b]furan-9-carbonitrile (14d). The product was prepared following the general procedure for photochemical reactions using 4-(2-(cyclohex-1-en-1-yl)ethoxy)-3-methoxybenzonitrile 10d (52 mg, 0.2 $\mathrm{mmol})$ and $\mathrm{MeCN}(110 \mathrm{~mL})$ distributed in 6 quartz glass tubes. The tubes were irradiated at a wavelength of $300 \mathrm{~nm}$ for 25 minutes. Column chromatography $\left(\mathrm{SiO}_{2}\right.$, petroleum ether/ethyl acetate $\left.=9: 1, \mathrm{KMnO}_{4} / \mathrm{UV}\right)$ afforded a mixture of the anti diastereomer $14 \mathrm{~d}(5 \mathrm{mg}, 10 \%)$ and the product $11 \mathbf{d}(2 \mathrm{mg}, 4 \%)$ as a colorless oil. The stereochemistry of the anti diastereomer 14d was resolved by 2D NMR NOESY experiment (cf ESI S35). Analytical data for the product $\mathbf{1 4 d}$ are reported for a mixture with $11 \mathbf{d}$. $R f=0.32$ (petroleum ether/ethyl acetate = 9:1, UV). ${ }^{1} \mathrm{H}$ NMR $\left(500 \mathrm{MHz}, \mathrm{CD}_{3} \mathrm{CN}\right): \delta 7.12(\mathrm{~s}, 1 \mathrm{H} ; \mathrm{CH}, 14 \mathrm{~d}), 5.86(\mathrm{~d}, J=5.9 \mathrm{~Hz}, 1 \mathrm{H}$; $\mathrm{CH}, 11 \mathrm{~d}), 5.78(\mathrm{~d}, J=5.9 \mathrm{~Hz}, 1 \mathrm{H} ; \mathrm{CH}, 11 \mathrm{~d}), 4.75(\mathrm{~d}, J=6.3, \mathrm{~Hz}, 1 \mathrm{H} ; \mathrm{CH}, 14 \mathrm{~d}), 4.13-4.10\left(\mathrm{~m}, 2 \mathrm{H} ; \mathrm{CH}_{2}, 14 \mathrm{~d}\right)$, $4.10-4.07\left(\mathrm{~m}, 1 \mathrm{H} ; \mathrm{CH}_{2}, 11 \mathrm{~d}\right), 3.96$ (ddd, $J=11.2,8.2,6.4 \mathrm{~Hz}, 1 \mathrm{H} ; \mathrm{CH}_{2}, 11 \mathrm{~d}$ ), 3.67 (d, J=6.3 Hz, $1 \mathrm{H} ; \mathrm{CH}$, 14d), 3.62 (s, 3H; $\mathrm{CH}_{3}, 11 \mathrm{~d}$ ), $3.26\left(\mathrm{~s}, 3 \mathrm{H} ; \mathrm{CH}_{3}, 14 \mathrm{~d}\right), 2.97$ (d, J = $\left.6.4 \mathrm{~Hz}, 1 \mathrm{H} ; \mathrm{CH}, 11 \mathrm{~d}\right), 2.48-2.43\left(\mathrm{~m}, 1 \mathrm{H} ; \mathrm{CH}_{2}\right.$, 14d), 2.37 (dd, $J=12.0,6.3 \mathrm{~Hz}, 1 \mathrm{H} ; \mathrm{CH}, 11 \mathrm{~d}$ ), 2.27 (ddd, $J=12.4,11.3,9.8 \mathrm{~Hz}, 1 \mathrm{H} ; \mathrm{CH}, 11 \mathrm{~d}$ ), 2.09 (ddt, $J=$ 13.6, 3.6, $\left.1.7 \mathrm{~Hz}, 1 \mathrm{H} ; \mathrm{CH}_{2}, 14 \mathrm{~d}\right), 1.88(\mathrm{~m}, 1 \mathrm{H} ; \mathrm{CH}, 14 \mathrm{~d}), 1.79-1.73\left(\mathrm{~m}, 1 \mathrm{H} ; \mathrm{CH}_{2}, 11 \mathrm{~d}\right), 1.70-1.65(\mathrm{~m}, 2 \mathrm{H}$; $\left.\mathrm{CH}_{2}, 14 \mathrm{~d}\right), 1.64\left(\mathrm{~m}, 3 \mathrm{H}, \mathrm{CH}_{2}, 11 \mathrm{~d}\right), 1.62-1.47\left(\mathrm{~m}, 5 \mathrm{H} ; \mathrm{CH}_{2}, 14 \mathrm{~d}\right), 1.50-1.42\left(\mathrm{~m}, 1 \mathrm{H} ; \mathrm{CH}_{2}, 11 \mathrm{~d}\right), 1.41-1.30$ (m, $\left.1 \mathrm{H} ; \mathrm{CH}_{2}, 14 \mathrm{~d}\right), 1.41-1.30\left(\mathrm{~m}, 2 \mathrm{H} ; \mathrm{CH}_{2}, 11 \mathrm{~d}\right), 1.29-1.17\left(\mathrm{~m}, 2 \mathrm{H} ; \mathrm{CH}_{2}, 11 \mathrm{~d}\right) .{ }^{13} \mathrm{C}\left\{{ }^{1} \mathrm{H}\right\} \mathrm{NMR}(126 \mathrm{MHz}$, $\left.\mathrm{CDCl}_{3}\right): \delta 168.1(\mathrm{Cq}, 14 \mathrm{~d}), 156.0(\mathrm{CH}, 14 \mathrm{~d}), 136.9(\mathrm{CH}, 11 \mathrm{~d}), 126.0(\mathrm{CH}, 11 \mathrm{~d}), 123.8(\mathrm{Cq}, 14 \mathrm{~d}), 119.6(\mathrm{CN}$, 11d), 114.5 (CN, 14d), 100.1 (Cq, 11d), 97.1 (Cq, 11d), 88.2 (CH, 14d), 87.4 (Cq, 14d), 69.3 ( $\left.\mathrm{CH}_{2}, 11 d\right), 68.9$ $\left(\mathrm{CH}_{2}, 14 \mathrm{~d}\right), 59.4(\mathrm{Cq}, 11 \mathrm{~d}), 57.6\left(\mathrm{CH}_{3}, 11 \mathrm{~d}\right), 54.0(\mathrm{CH}, 11 \mathrm{~d}), 52.3\left(\mathrm{CH}_{3}, 14 \mathrm{~d}\right), 49.8(\mathrm{CH}, 14 \mathrm{~d}), 47.5(\mathrm{CH}, 14 \mathrm{~d})$, 44.4 (Cq, 14d), 41.1 ( $\left.\mathrm{CH}_{2}, 11 \mathrm{~d}\right), 39.7(\mathrm{CH}, 11 \mathrm{~d}), 36.7\left(\mathrm{CH}_{2}, 14 \mathrm{~d}\right), 31.1\left(\mathrm{CH}_{2}, 11 \mathrm{~d}\right), 30.1(\mathrm{Cq}, 11 \mathrm{~d}), 27.9\left(\mathrm{CH}_{2}\right.$, 14d), $27.3\left(\mathrm{CH}_{2}, 11 \mathrm{~d}\right), 24.0\left(\mathrm{CH}_{2}, 11 \mathrm{~d}\right), 23.8\left(\mathrm{CH}_{2}, 14 \mathrm{~d}\right), 22.9\left(\mathrm{CH}_{2}, 14 \mathrm{~d}\right), 22.7\left(\mathrm{CH}_{2}, 11 \mathrm{~d}\right), 21.8\left(\mathrm{CH}_{2}, 14 \mathrm{~d}\right) . \mathrm{IR}$ $v\left(\mathrm{~cm}^{-1}\right)=2935,2225,942,884,807,704,680$. HRMS (ESI) $: \mathrm{m} / z[\mathrm{M}+\mathrm{H}]^{+}$calcd for $\left[\mathrm{C}_{16} \mathrm{H}_{20} \mathrm{NO}_{2}\right]^{+}: 258.1494$, found : 258.1497.

3-allyl-4-hydroxy-5-methoxybenzonitrile (15). The product was prepared following the general procedure for photochemical reactions using 4-(allyloxy)-3-methoxybenzonitrile $10 \mathrm{~g}$ (51 mg, 0.27 
$\mathrm{mmol}$ ) and $\mathrm{MeCN}(15 \mathrm{~mL})$. The tube was irradiated at a wavelength of $300 \mathrm{~nm}$ for $4 \mathrm{~h}$. Column chromatography $\left(\mathrm{SiO}_{2}\right.$, petroleum ether/ethyl acetate $\left.=8: 2, \mathrm{KMnO}_{4}\right)$ afforded the product $15(19 \mathrm{mg}, 37$ \%) as a colorless oil. $R f=0.21$ (petroleum ether/ethyl acetate $=9: 1, \mathrm{UV}) .{ }^{1} \mathrm{H} \mathrm{NMR}\left(500 \mathrm{MHz}, \mathrm{CDCl}_{3}\right): \delta 7.12$ (d, J = 1.6 Hz, 1H; $\operatorname{Ar}-\mathrm{H}$ ), 6.98 (d, J = 1.8 Hz, 1H; $\operatorname{Ar}-\mathrm{H}$ ), 6.15 (s, 1H; OH), 5.94 (ddt, J = 16.8, 10.2, $6.6 \mathrm{~Hz}, 1 \mathrm{H}$; $\mathrm{CH}), 5.21-5.03\left(\mathrm{~m}, 2 \mathrm{H} ; \mathrm{CH}_{2}\right), 3.92\left(\mathrm{~s}, 3 \mathrm{H} ; \mathrm{CH}_{3}\right), 3.40\left(\mathrm{dd}, J=6.6,1.5 \mathrm{~Hz}, 2 \mathrm{H} ; \mathrm{CH}_{2}\right) .{ }^{13} \mathrm{C}\left\{{ }^{1} \mathrm{H}\right\} \mathrm{NMR}(126 \mathrm{MHz}$, $\mathrm{CDCl}_{3}$ ): $\delta 147.7(\mathrm{Cq}), 146.4(\mathrm{Cq}), 135.1(\mathrm{CH}), 127.6(\mathrm{CH}), 127.3(\mathrm{Cq}), 119.6(\mathrm{CN}), 116.9\left(\mathrm{CH}_{2}\right), 111.9(\mathrm{CH})$, $102.8(\mathrm{Cq}), 56.5\left(\mathrm{CH}_{3}\right), 33.4\left(\mathrm{CH}_{2}\right) . \mathrm{IR} \vee\left(\mathrm{cm}^{-1}\right)=2977,2222,1639,1598,915,785 . \mathrm{HRMS}(\mathrm{ESI}): \mathrm{m} / z[\mathrm{M}+\mathrm{H}]^{+}$ calcd for $\left[\mathrm{C}_{11} \mathrm{H}_{12} \mathrm{NO}_{2}\right]^{+}: 190.0868$, found : 190.0869 .

\section{8-methoxy-3,3a,4,6a-tetrahydro-2H-cyclobuta[h]benzofuran-6a-carbonitrile (17a).}

The product was prepared following the general procedure for photochemical reactions using 4-(but-3en-1-yloxy)-3-methoxybenzonitrile 10a (60 mg, $0.30 \mathrm{mmol}), \mathrm{MeCN}(10 \mathrm{~mL})$ and acetone $(20 \mathrm{~mL})$. The tube was irradiated at a wavelength of $300 \mathrm{~nm}$ for $8 \mathrm{~h}$. Column chromatography $\left(\mathrm{SiO}_{2}\right.$, petroleum ether/ethyl acetate $\left.=9: 1, \mathrm{KMnO}_{4} / \mathrm{UV}\right)$ afforded the products $17 \mathrm{a}(7 \mathrm{mg}, 12 \%) . R f=0.5$ (petroleum ether/ethyl acetate = 9:1, UV). ${ }^{1} \mathrm{H} N M R\left(500 \mathrm{MHz}, \mathrm{CDCl}_{3}\right): \delta 5.93(\mathrm{~s}, 2 \mathrm{H} ; 2 \mathrm{CH}), 4.96(\mathrm{~s}, 1 \mathrm{H} ; \mathrm{CH}), 4.29\left(\mathrm{t}, J=8.6 \mathrm{~Hz}, 1 \mathrm{H} ; \mathrm{CH}_{2}\right), 4.23$ $-4.11\left(\mathrm{~m}, 1 \mathrm{H} ; \mathrm{CH}_{2}\right), 3.62\left(\mathrm{~s}, 3 \mathrm{H} ; \mathrm{CH}_{3}\right), 2.32\left(\mathrm{dt}, \mathrm{J}=13.5,3.9 \mathrm{~Hz}, 1 \mathrm{H} ; \mathrm{CH}_{2}\right), 2.15-1.90\left(\mathrm{~m}, 3 \mathrm{H} ; \mathrm{CH}_{2}, \mathrm{CH}\right), 1.82$ (dd, $J=16.4,12.5 \mathrm{~Hz}, 1 \mathrm{H} ; \mathrm{CH}) .{ }^{13} \mathrm{C}\left\{{ }^{1} \mathrm{H}\right\} \operatorname{NMR}\left(126 \mathrm{MHz}, \mathrm{CDCl}_{3}\right): \delta 159.3(\mathrm{Cq}), 131.0(\mathrm{CH}), 128.7(\mathrm{CH}), 119.7$ (CN), $102.7(\mathrm{CH}), 90.8(\mathrm{Cq}), 70.2\left(\mathrm{CH}_{2}\right), 56.5(\mathrm{CH}), 43.8(\mathrm{CH}), 41.2(\mathrm{Cq}), 29.8(\mathrm{Cq}), 27.8\left(\mathrm{CH}_{2}\right), 25.5\left(\mathrm{CH}_{2}\right) . \mathrm{IR}$ $v\left(\mathrm{~cm}^{-1}\right)=3094,2942,2231,1647,914,790$. HRMS (ESI) $: \mathrm{m} / z[\mathrm{M}+\mathrm{H}]^{+}$calcd for $\left[\mathrm{C}_{12} \mathrm{H}_{14} \mathrm{NO}_{2}\right]^{+}: 204.1025$, found : 204.1025 .

\section{4a-methoxy-4,4-dimethyl-2,3,4,4a,6a,7-hexahydrocyclobuta[f]benzo-furan-6-}

carbonitrile (18). When the mixture of diastereomers $\mathbf{1 4 c}$ and $\mathbf{1 4 c ^ { \prime }}$ was left overnight in NMR solvent $\mathrm{CDCl}_{3}$, syn diastereomer $14 \mathrm{c}$ disappeared and two new products formed. One product (18) seemed to be the result of a 1,3-H-shift in the cyclohexene moiety.

Analytical data are reported for the mixture of 18 , anti diastereomer $14 \mathrm{c}^{\prime}$ and the unknown product. ${ }^{1} \mathrm{H}$ NMR (500 MHz, CDCl 3 ): $\delta 6.81\left(\mathrm{~s}, 1 \mathrm{H} ; \mathrm{CH}, 14 \mathrm{c}^{\prime}\right), 6.80$ (d, J = $1.0 \mathrm{~Hz}, 1 \mathrm{H} ; \mathrm{CH}$, unknown), $6.74(\mathrm{~s}, 1 \mathrm{H} ; \mathrm{CH}, 18)$, $4.82\left(\mathrm{dd}, J=6.3,2.6 \mathrm{~Hz}, 1 \mathrm{H} ; \mathrm{CH}, 14 \mathrm{c}^{\prime}\right), 4.32$ (dd, J = 10.2, 8.4 Hz, 2H; $\left.\mathrm{CH}_{2}, 18\right), 4.24(\mathrm{td}, J=8.5,1.9 \mathrm{~Hz}, 1 \mathrm{H}$; $\left.\mathrm{CH}_{2}, 14 \mathrm{c}^{\prime}\right), 4.03-3.94\left(\mathrm{~m}, 1 \mathrm{H} ; \mathrm{CH}_{2}, 14 \mathrm{c}^{\prime}\right), 3.97-3.89\left(\mathrm{~m}, 1 \mathrm{H} ; \mathrm{CH}_{2}\right.$, unknown), $3.68(\mathrm{~d}, J=6.3 \mathrm{~Hz}, 1 \mathrm{H} ; \mathrm{CH}$, $\left.14 c^{\prime}\right), 3.48(\mathrm{dd}, J=6.3,1.3 \mathrm{~Hz}, 1 \mathrm{H} ; \mathrm{CH}, 18), 3.42-3.34\left(\mathrm{~m}, 1 \mathrm{H} ; \mathrm{CH}\right.$, unknown), $3.35\left(\mathrm{~s}, 3 \mathrm{H} ; \mathrm{CH}_{3}, 18\right), 3.33$ (s, $3 \mathrm{H} ; \mathrm{CH}_{3}$, unknown), 3.32 (s, 3H; $\mathrm{CH}_{3}, 14 \mathrm{c}^{\prime}$ ), $2.65-2.56\left(\mathrm{~m}, 2 \mathrm{H} ; \mathrm{CH}_{2}, 18\right), 2.42$ (ddd, J = 11.6, 9.0, $2.8 \mathrm{~Hz}, 1 \mathrm{H}$; $\left.\mathrm{CH}, 14 c^{\prime}\right), 2.38\left(\mathrm{~m}, 1 \mathrm{H} ; \mathrm{CH}_{2}, 18\right), 2.36\left(\mathrm{~m}, 1 \mathrm{H} ; \mathrm{CH}_{2}\right.$, unknown), $2.27\left(\mathrm{~m}, 1 \mathrm{H} ; \mathrm{CH}_{2}, 18\right), 2.21-2.16\left(\mathrm{~m}, 1 \mathrm{H} ; \mathrm{CH}_{2}\right.$, unknown), 2.15 (d, J = 5.0 Hz, 1H; CH, unknown), 2.07 (m, 1H; $\mathrm{CH}_{2}$, unknown), $1.99\left(\mathrm{~m}, 1 \mathrm{H} ; \mathrm{CH}_{2}, 14 \mathrm{c}^{\prime}\right), 1.93$ 
(m, $\left.1 \mathrm{H} ; \mathrm{CH}_{2}, 14 \mathrm{c}^{\prime}\right), 1.83$ (ddt, J=12.8, 8.6, $4.5 \mathrm{~Hz}, 1 \mathrm{H} ; \mathrm{CH}_{2}$, unknown), 1.05 (s, 3H; $\left.\mathrm{CH}_{3}, 18\right), 1.05$ (s, 3H; $\mathrm{CH}_{3}$, 18), 1.04 (s, 3H; $\left.\mathrm{CH}_{3}, 14 \mathrm{c}^{\prime}\right), 1.00$ (s, 3H; $\left.\mathrm{CH}_{3}, 18\right), 0.94$ (s, 3H; $\mathrm{CH}_{3}$, unknown), 0.90 ( $\mathrm{s}, 3 \mathrm{H} ; \mathrm{CH}_{3}$, unknown), $0.84\left(\mathrm{~s}, 3 \mathrm{H} ; \mathrm{CH}_{3}, 14 \mathrm{c}^{\prime}\right) .{ }^{13} \mathrm{C}\left\{{ }^{1} \mathrm{H}\right\} \mathrm{NMR}\left(126 \mathrm{MHz}, \mathrm{CDCl}_{3}\right): \delta 161.10$ (Cq, 14c') $152.29(\mathrm{CH}, 18), 152.02(\mathrm{CH}$, unknown), 149.60 (CH, 14c'), 146.83 (Cq, 18), 124.00 (CN, 6c), 120.63 (CN, 18), 120.46 (CN, 14c'), 113.37 $\left(\mathrm{Cq}, 14 c^{\prime}\right), 113.11$ (Cq, unknown), 112.66 (Cq, 18), 111.86 (Cq, 18), 104.51 (Cq, unknown), 90.09 (Cq, 14c'), $88.90(\mathrm{Cq}, 18), 88.02$ ( $\mathrm{Cq}$, unknown), $86.30\left(\mathrm{CH}, 14 \mathrm{c}^{\prime}\right), 70.56\left(\mathrm{CH}_{2}, 14 \mathrm{c}^{\prime}\right), 69.93\left(\mathrm{CH}_{2}, 18\right), 65.77\left(\mathrm{CH}_{2}\right.$, unknown), $52.85\left(\mathrm{CH}_{3}, 18\right), 52.39\left(\mathrm{CH}_{3}, 14 \mathrm{c}^{\prime}\right), 52.09\left(\mathrm{CH}_{3}\right.$, unknown), $51.33\left(\mathrm{CH}\right.$, unknown), $47.86\left(\mathrm{CH}, 14 c^{\prime}\right)$, 47.05 ( $\mathrm{CH}, 18), 45.91$ ( $\mathrm{CH}$, unknown), $45.37\left(\mathrm{CH}, 14 \mathrm{c}^{\prime}\right), 40.25$ (Cq, 14c'), 39.40 (Cq, unknown), 37.74 (Cq, 18), 37.39 ( $\mathrm{CH}_{2}$, unknown), $29.62\left(\mathrm{CH}_{2}, 18\right), 25.46\left(\mathrm{CH}_{2}\right.$, unknown), $25.12\left(\mathrm{CH}_{2}, 14 c^{\prime}\right), 24.33\left(\mathrm{CH}_{3}\right.$, unknown), $22.98\left(\mathrm{CH}_{2}, 18\right), 22.98\left(\mathrm{CH}_{3}, 18\right), 22.57\left(\mathrm{CH}_{3}, 18\right), 22.41\left(\mathrm{CH}_{3}, 14 c^{\prime}\right), 16.49\left(\mathrm{CH}_{3}\right.$, unknown $), 14.22\left(\mathrm{CH}_{3}, 14 c^{\prime}\right)$. IR $\vee\left(\mathrm{cm}^{-1}\right)=2972,2950,2218,1679,989,942,876,800,789,711$. HRMS (ESI) $: \mathrm{m} / \mathrm{z}[\mathrm{M}+\mathrm{H}]^{+}$calcd for $\left[\mathrm{C}_{14} \mathrm{H}_{18} \mathrm{NO}_{2}\right]^{+}: 232.1338$, found : 232.1337.

Supporting information. ${ }^{1} \mathrm{H}$ and ${ }^{13} \mathrm{C}\left\{{ }^{1} \mathrm{H}\right\}$ NMR spectra for all compounds, ${ }^{19} \mathrm{~F}$ NMR spectra for compounds $10 \mathrm{e}, 10 \mathrm{i}, 11 \mathrm{e}, 12 \mathrm{e}$, configuration assignments of $14 \mathrm{c}, 14 c^{\prime}, 14 \mathrm{~d}$ by NOESY experiment, UV absorption spectra of compounds 5, 10a, 10f, 11a, 12a and X-ray structures of compounds 11a, 11b, 11c, 11e, 13c.

\section{Acknowledgments}

We are grateful to the Communauté Urbaine du Grand Reims and the Université de Reims ChampagneArdenne.

\section{References}

\footnotetext{
${ }^{1}$ (a) Klán, P.; Wirz, J. Photochemistry of Organic Compounds. Wiley, Chichester, 2009. (b) Buzzetti, L.; Crisenza, G. E. M.; Melchiorre, P. Mechanistic Studies in Photocatalysis. Angew. Chem. Int. Ed. 2019, 58, 3730-3747.

${ }^{2}$ (a) Turro, N. J. Geometric and Topological Thinking in Organic Chemistry. Angew. Chem. Int. Ed. 1986, 25, 882-901. (b) Turro, N. J.; Schuster, G. Photochemical Reactions as a Tool in Organic Synthesis. Science 1975, 187, 303-312. (c) Zimmerman, H. E. Mechanistic Organic Photochemistry. Angew. Chem. Int. Ed. $1969,8,1-11$.
} 
${ }^{3}$ (a) Hoffmann. N. Photochemical Reactions as Key Steps in Organic Synthesis. Chem. Rev. 2008, 108, 1052-1103. (b) Bach, T.; Hehn, J. P. Photochemical Key Steps in Natural Product Synthesis. Angew. Chem. Int. Ed. 2011, 50, 1000-1045. (c) Beeler, A. B., Ed. Photochemistry in Organic Synthesis [Special issue]. Chem. Rev. 2016, 116 (17).

${ }^{4}$ Bonfield, H.; Knauber, T.; Lévesque, F.; Moschetta, E. G.; Susanne, F.; Edwards, L. J. Photons as a 21st century reagent. Nat. Commun. 2020, 11, 804

${ }^{5}$ (a) Rosenberg, M.; Dahlstrand, C.; Kilså, K.; Ottosson, H. Excited State Aromaticity and Antiaromaticity: Opportunities for Photophysical and Photochemical Rationalization. Chem. Rev. 2014, 114, 5379-5425. (b) Salina, T.; Ayub, R.; Toldo, J.; Sundell, J.; Rabten, W.; Nicaso, M.; Alabugin, I.; Galván, I. F.; Gupta, A. K.; Linth, R.; Othaber, A.; Lewis, R. J.; Grönberg, G.; Bergman, J.; Ottosson, H. Impact of Excited-State Antiaromaticity Relief in a Fundamental Benzene Photoreaction Leading to Substituted Bicyclo[3.1.0]hexenes. J. Am. Chem. Soc. 2020, 142, 10942-10954. (c) Papadakis, R.; Li, H.; Bergman, J.; Lundstedt, A.; Jorner, K.; Ayub, R.; Haldar, S.; Jahn, B. O.; Denisova, A.; Zietz, B.; Linth, R.; Sanjal, B.; Grennberg, H.; Leifer, K.; Ottosson, H. Metal-free photochemical silylations and transfer hydrogenations of benzenoid hydrocarbons and graphene. Nat. Commun. 2016, 7, 12962.

${ }^{6}$ Hoffmann, N. Photochemical reactions of aromatic compounds and the concept of the photon as a traceless reagent. Photochem. Photobiol. Sci. 2012, 11, 1613-1641.

${ }^{7}$ Cornelisse. J. The Meta Photocycloaddition of Arenes to Alkenes. Chem. Rev. 1993, 93, 615-669.

${ }^{8}$ Cornelisse, J.; de Haan, R. Ortho Photocycloaddition of Alkenes and Alkynes to the Benzene Ring. In Understanding and Manipulating Excited-State Processes (Ramamurthy, V.; Schanze, K. S.; Eds.), Marcel Dekker, New York, 2001, pp. 1-126.

${ }^{9}$ (a) Hoffmann, N. Photochemical Cycloaddition between Benzene Derivatives and Alkenes. Synthesis 2004, 481-495. (b) Remy, R.; Bochet, C. G. Arene-Alkene Cycloaddition. Chem. Rev. 2016, 116, 98169849.

${ }^{10}$ (a) Wender, P. A. Arene-Alkene Photocycloaddition reactions. Org. Photochem. 1989, 10, 357-473. (b) De Keukeleire, D.; He, S.-H. Photochemical Strategies for the Construction of Polycyclic Molecules. Chem. Rev. 1993, 93, 559-380. (c) Müller, F.; Mattay, J. Photocycloadditions: Control by Energy and Electron Transfer. Chem. Rev. 1993, 93, 99-117. (d) Wender, P. A.; Dore, T. M. Intra- and Intermolecular Cycloadditions of Benzene Derivatives. In CRC Handbook of Organic Photochemistry and Photobiology (Horspool, W. M.; Song, P.-S.; Eds.), CRC Press, Boca Raton, 1995, pp. 280-290. (e) Gilbert, A. Intra- and Intermolecular Cycloadditions of Benzene Derivatives. In CRC Handbook of Photochemistry and Photobiology, 2nd Ed. (Horspool, W.; Lenci, F.; Eds.), CRC Press, Boca Raton, 2004, pp. 41/1-41/11. (f) 
Gaich, T. The Arene-Alkene Photocycloaddition. In Comprehensive Organic Synthesis II, Vol. 5 (Knochel, P.; Ed.), Elsevier, Amsterdam, 2014, pp. 703-731.

${ }^{11}$ For some reviews see: (a) Roche, S. P.; Porco Jr., J. A. Dearomatization Strategies in the Synthesis of Complex Natural Products. Angew. Chem. Int. Ed. 2011, 50, 4068-4093. (b) Wertjes, W. C.; Southgate, E. H.; Sarlah, D. Recent advances in chemical dearomatization of nonactivated arenes. Chem. Soc. Rev. 2018, 47, 7996-8017. (c) López Ortiz, F.; José Iglesias, M.; Fernández, I.; Andújar Sánchez, C. M.; Ruiz Gómez, G. Nucleophilic Dearomatization ( $\left.D_{N} A R\right)$ Reactions of Aromatic C,H-Systems. A Mature Paradigm in Organic Synthesis. Chem. Rev. 2007, 107, 1580-1691.

${ }^{12}$ (a) Oelgemöller, M.; Jung, C.; Mattay, J. Green photochemistry: production of fine chemicals with sunlight. Pure Appl. Chem. 2007, 79, 1939-1947. (b) Lefebvre, C.; Hoffmann, N. Photochemical rearrangements in organic synthesis and the concept of the photon as a traceless reagent. In Nontraditional Activation Methods in Green and Sustainable Applications (Török, B.; Schäfer, C.; Eds.), Elsevier, Amsterdam, 2021, pp 283-328.

${ }^{13}$ (a) Gallezot. P. Conversion of biomass to selected chemical products. Chem. Soc. Rev. 2012, 41, 15381558. (b) Tuck, C. O.; Pérez, E.; Horvath, I.; Sheldon, R. A.; Poliakoff, M. Valorization of Biomass: Deriving More Value from Waste. Science 2012, 337, 695-699. (c) Ravelli, D.; Samorì, C. (Eds.) Biomass Valorisation, Wiley-VCH, Weinheim, 2021.

${ }^{14}$ Heitner, C.; Dimmel, D. R.; Schmidt, J. A.; Eds., Lignin and Lignans, CRC Press, Boca Raton, 2010. ${ }^{15}$ (a) Beckham, G. T.; Ed. Lignin Valorization, Energy and Environment Series No. 19, Royal Society of Chemistry, 2018. (b) Zakzeski, J.; Bruijnincx, P. C. A.; Jongerius, A. L.; Weckhuysen, B. W. The Catalytical Valorization of Lignin for the Production of Renewable Chemicals. Chem. Rev. 2014, 114, 3552-3599. (c) Ragauskas, A. J.; Beckham, G. T.; Biddy, M. J.; Chandra, R.; Chen, F.; Davis, M. F.; Davison, B. H.; Dixon, R. A.; Gilna, P.; Keller, M.; Langan, P.; Naskar, A. K.; Saddler, J. N.; Tschaplinski, T. J.; Tuskan, G. A.; Wyman, C. E. Lignin Valorization: Improving Lignin Processing in the Biorefinery. Science 2014, 344, 1246843.

${ }^{16}$ Fache, M.; Boutevin, B.; Caillol, S. Vanillin Production from Lignin and Its Use as a Renewable Chemical. ACS Stustainable Chem. Eng. 2016, 4, 35-46. Araújo, J. D. P.; Grande, C. A.; Rodrigues, A. E. Vanillin production from lignin oxidation in a batch reactor. Chem. Eng. Res. Des. 2010, 88, 1024-1032. Mota, M. I. F. P.; Rodrigues Pinto, C.; Loureiro, J. M.; Rodrigues, A. E. Recovery of Vanillin and Syringaldehyde from Lignin Oxidation: A Review of Separation and Purification Processes. Sep. Purif. Rev. 2016, 45, 227-259. Fache, M.; Darroman, E.; Besse, V.; Auvergne, R.; Caillol, S.; Boutevin, B. Vanillin, a promising biobased building-block for monomer synthesis. Green Chem. 2014, 16, 1987-1998.

${ }^{17}$ (a) De Keukeleire, D. The Synthetic Potential of the Intramolecular metha-Photocycloaddition in Arenealkene Bichromophoric Systems Containing Oxygen in the Tether. Aldrichchimica Acta 1994, 27, 59-69. 
(b) De Keukeleire, D.; He, S.-L.; Blakemore, D.; Gilbert, A. Intramolecular photocycloaddition reactions of 4-phenoxybut-1-enes. J. Photochem. Photobiol. A 1994, 80, 233-240. (c) Vízvárdi, K.; Toppet, S.; Hoornaert, G. J.; De Keukeleire, D.; Bako, P.; Van der Eycken, E. Intramolecular ortho and meta photocycloadditions of 4-phenoxybut-1-enes substituted in the arene residue with carbomethoxy, carbomethoxymethyl, and 2-carboxymethoxyethyl groups. J. Photochem. Photobiol., A 2000, 133, 135146.

${ }^{18}$ (a) Bryce-Smith, D.; Gilbert, A. The organic photochemistry of benzene - I. Tetrahedron 1976, 32, 13091326. (b) Bryce-Smith, D.; Gilbert, A. The organic photochemistry of benzene - II. Tetrahedron 1977, 33, 2459-2489.

${ }^{19}$ (a) Hoffmann, N.; Pete, J.-P. Intramolecular Photochemical Reactions of Bichromophoric 2(Alkenyloxy)phenols and 1-(Alkenyloxy)-3-(alkyloxy)benzene Derivatives. Acid-Catalyzed Transformations of the Primary Cycloadducts. J. Org. Chem. 1997, 62, 6952-6960. (b) Verrat, C.; Hoffmann, N.; Pete, J.-P. An Easy Access to Benzo[f]isoquinoline Derivatives Using Benzocyclobutenes Derived from Resorcinol. Synlett 2000, 1166-1168.

${ }^{20}$ Hoffmann, N.; Pete, J. P. Intramolecular Photochemical Reactivity of O-Alk-3-enylsalicylic Esters. Tetrahedron Lett. 1995, 36, 2623-2626.

${ }^{21}$ Hoffmann, N.; Pete, J.-P. Intramolecular [2+2] Photocycloaddition of Bichromophoric Derivatives of 3,5-Dihydroxybenzoic Acid and 3,5-Dihydroxybenzonitrile. Synthesis 2001, 1236-1242.

${ }^{22}$ (a) Hoffmann, N.; Pete, J.-P.; Inoue, Y.; Mori, T. Novel [2+2] Photocycloaddition-Induced Rearrangement of Bichromophoric Naphthalene-Tethered Resorcinol Ethers. J. Org. Chem. 2002, 67, 2315-2322. (b) Hoffmann, N. New photochemical rearrangements and extrusion reactions of aromatic compounds induced by an intramolecular [2+2] photocycloaddition between a naphthalene and a resorcinol moiety. Tetrahedron 2002, 58, 7933-7941.

${ }^{23}$ (a) Kalena, G. P.; Pradhan, P.; Banerji, A. Stereo- and regioselectivity of intramolecular 1,2-arenealkene photocycloaddition in 2-alkene-4-chromanones. Tetrahedron 1999, 55, 3209-3218. (b) Howell, N.; Pincock, J. A.; Stefanova, R. The Phototransposition in Acetonitrile and the Photoaddition of 2,2,2Trifluoroethanol to the Six Isomers of Dimethylbenzonitrile. J. Org. Chem. 2000, 65, 6173-6178. (c) Mori, T.; Wada, T.; Inoue, Y. Perfect Switching of Photoreactivity by Acid: Photochemical Decarboxylation versus Transesterification of Mesityl Cyclohexanecarboxylate. Org. Lett. 2000, 21, 3401-3404.

${ }^{24}$ Wagner, P. J. Photoinduced Ortho [2+2] Cycloaddition as Double Bonds to Triplet Benzenes. Acc. Chem. Res. 2001, 34, 1-8.

${ }^{25}$ For recent examples see: (a) Zech, A.; Bach, T. Photochemical Reaction Cascade from O-Pent-4-enylSubstituted Salicylates to Complex Multifunctional Scaffolds. J. Org. Chem. 2018, 83, 3069-3077. (b) 
Næsborg, L.; Jandl, C.; Zech, A.; Bach, T. Complex Carbocyclic Skeleton from Aryl Ketones through a Three-Photon Cascade Reaction. Angew. Chem. Int. Ed. 2020, 59, 5656-5659. (c) Zech, A.; Jandl, C.; Bach, T. Concise Access to the Skeleton of Protoilludane Sesquiterpenes through a Photochemical Reaction Cascade: Total Synthesis of Atlanticone C. Angew. Chem. Int. Ed. 2019, 58, 14629-1432. (d) Proessdorf, J.; Zech, A.; Jandl, C.; Bach, T. Concice Total Synthesis of (+)-Atlanticone C. Synlett 2020, 31, 1598-1602.

${ }^{26}$ Al-Qaradawi, S. Y.; Cosstick, K. B.; Gilbert, A. Intramolecular Photocycloaddition of 4-Phenoxybut-1enes: a Convenient Access to the 4-Oxatricyclo[7.2.0.0 3,7 undeca-2,10-diene Skeleton. J. Chem. Soc. Perkin Trans. I 1992, 1145-1148.

${ }^{27}$ Al-Jalal, N.; Gilbert, A. Substituent effects on the photocycloaddition reactions of anisoles to acrylonitriles. Recl. Tav. Chim. Pays-Bas 1990, 109, 21-25.

${ }^{28}$ Thompson, R.; Doggrell, S.; Hoberg, J. O. Potassium Channel Activators Based on the Benzopyran Substructure: Synthesis and Activity of the C-8 Substituent. Bioorg. Med. Chem. 2003, 11, 1663-1668.

${ }^{29}$ Lipshutz, B. H.; Ghorai, S.; Leong, W. W. Y. Deprotection of Homoallyl (hAllyl) Derivatives of Phenols, Alcohols, Acids, and Amines. J. Org. Chem. 2009, 74, 2854-2857.

${ }^{30}$ For similar examples of a vinyl cyclopropane rearrangement see: (a) Wender, P. A.; Dore, T. M. A Formal Synthesis of Crinipellin B Based on the Arene-Alkene meta-Photocycloaddition Reaction. Tetrahedron Lett. 1998, 39, 8589-8592. (b) Wender, P. A.; deLong, M. A. Synthetic Studies of AreneOlefin Cycloadditions. XII. Total Synthesis of (+/-)-Subergoric Acid. Tetrahedron Lett. 1990, 31, 54295432. (c) Wender, P. A.; Dreyer, G. B. Synthetic Studies on Arene-Olefin Cycloadditions. II. Total Synthesis of (+/-)-Isocumene. Tetrahedron 1981, 37, 4445-4450.

${ }^{31}$ Galino, F. The photochemical rearrangement of aromatic ethers - A review of the Photo-Claisen reaction, J. Photochem. Photobiol. C 2005, 6, 123-138.

${ }^{32}$ Wagner, P. J.; Sakamoto, M.; Madkour, A. E. Regioselectivity in Intramolecular Cycloaddition of Double Bonds to Triplet Benzenes. J. Am. Chem. Soc. 1992, 114, 7298-7299.

${ }^{33}$ Wagner, P. J.; Smart, R. P. Acetone Sensitized Intramolecular ortho Photocyclization of Substituted 4Phenoxybut-1-enes.Tetrahedron Lett. 1995, 36, 5135-5138.

${ }^{34}$ Magano, J.; Chen, M. H.; Clark, J. D.; Nussbaumer, T. 2-(Diethylamino)ethanethiol, a New Reagent for the Odorless Deprotection of Aromatic Methyl Ethers. J. Org. Chem. 2006, 71, 7103-7105.

${ }^{35}$ Ghosh, A. K.; Nickponski, D. R. Cu(II)-Catalyzed Olefin Migration and Prins Cyclization: Highly Diastereoselective Synthesis of Substituted Tetrahydropyrans. Org. Lett. 2011, 13, 4328-4331.

${ }^{36}$ Belleau, B.; Gulini, U.; Gour-Salin, B.; Ahmed, F. R. Synthesis and crystal structure of 17-deaza-17-methyl thionium isomorphinan (isosulforphanol) perchlorate, an isostere of the opiate isolevorphanol. Can. J. Chem. 1985, 63, 1268-1274. 
${ }^{37}$ Sheldrick, G. M. Program for Crystal Structure Solution; University of Göttingen: Göttingen, Germany, 1997.

${ }^{38}$ Sun, X.; Chen, J.; Ritter, T. Catalytic dehydrogenative decarboxyolefination of carboxylic acids. Nat. Chem. 2018, 10, 1229-1233. 